\title{
The Fate of Dicationic States in Molecular Clusters of Benzene and Related Compounds
}

by

\author{
Michael S. Deleuze, Jean-Pierre François and Eugene S. Kryachko
}

\section{SUPPORTING INFORMATION}

\section{APPENDIX I : Preliminary Study of Larger and/or Hybrid Dicationic Assemblies of Benzene, Hexafluorobenzene and Naphthalene}

Prior to performing complete but also computationally very demanding investigations of reaction paths, the main goal of the preliminary calculations presented here is simply to investigate the molecular structure of large dicationic and covalently bound assemblies containing three benzene (Figure 7) or hexafluorobenzene (Figure 8) rings, or two naphthalene units (Figure 9) using the B3LYP/6-31+G(d,p) approaches. Larger and/or hybrid dicationic assemblies containing benzene and naphthalene moieties have also been studied using the computationally simpler Hartree-Fock (HF) approach, along with the 6$31 \mathrm{G}$ basis set (Figures 10-12). We refer to Appendix II for the optimized Cartesian coordinates and the corresponding energies of these structures.

Two stable structures could be identified from B3LYP/6-31+G(d,p) calculations for the benzene trimer dication, $\mathrm{Bz}_{3}{ }^{2+}-\mathrm{I}$ and $\mathrm{Bz}_{3}{ }^{2+}$-II (Figure 7). The former species has been simply obtained by optimizing the geometry of a cluster containing the $\mathrm{Bz}_{2}{ }^{2+}$-I species and an additional benzene molecule nearby as input structure. This readily results into the formation of a second inter-ring $\mathrm{C}-\mathrm{C}$ bond, a fact which confirms the suggestion that further charge transfer reactions in larger molecular clusters are possible. As Figure 7 shows, these bonds are stretched by $0.025 \AA$ compared to the $\mathrm{C}_{4}-\mathrm{C}_{7}$ bond in $\mathrm{Bz}_{2}{ }^{2+}$-I (Figure 4), which reflects their weakening with increasing system size, despite a release of Coulomb repulsions with a repositioning of the two holes on the four $\mathrm{C}-\mathrm{H}$ bonds embracing the two fusion bonds. Two of the $\mathrm{C}-\mathrm{H}$ bonds that belong to the middle benzene molecule are therefore contracted by $0.02 \AA$ only, due to the 
enhanced charge delocalization. In straightforward analogy with Peierls distortion effects in low bandgap (conducting) polymers (see ref. 49 and references therein), this weakening of the inter-ring C-C bonds may be ascribed to a significant decrease of the HOMO-LUMO band gap, from $4.09 \mathrm{eV}$ for $\mathrm{Bz}_{2}{ }^{2+}$ I, to $3.1 \mathrm{eV}$ for $\mathrm{Bz}_{3}{ }^{2+}-\mathrm{I}$, and an enhanced propensity therefore to exhibit longer bonds between atoms that connect different monomers, which helps preventing the closure of the band gap between occupied and unoccupied levels and near energy degeneracies therefore. The $\mathrm{Bz}_{3}{ }^{2+}-\mathrm{II}$ complex, which differs from the $\mathrm{Bz}_{3}{ }^{2+}$-I species by the transfer of two protons from the $\left\{\mathrm{C}_{1}, \mathrm{C}_{4}\right\}$ to the $\left\{\mathrm{C}_{2}, \mathrm{C}_{5}\right\}$ positions in the central ring, where they form two $\mathrm{CH}_{2}$ groups lying at opposite sites in the ring, is found at $5.8 \mathrm{kcal} / \mathrm{mol}$ below the latter one. This was expected since the central ring partly resembles that in $\mathrm{Bz}_{2}{ }^{2+}-\mathrm{II}$, a complex which is located at $8.7 \mathrm{kcal} / \mathrm{mol}$ below $\mathrm{Bz}_{2}{ }^{2+}-\mathrm{I}$ (Section 3). In line with the release of Coulomb repulsions between rings, the fusion bonds in $\mathrm{Bz}_{3}{ }^{2+}-\mathrm{II}$ are therefore much shorter than those seen in $\mathrm{Bz}_{3}{ }^{2+}-\mathrm{I}$.

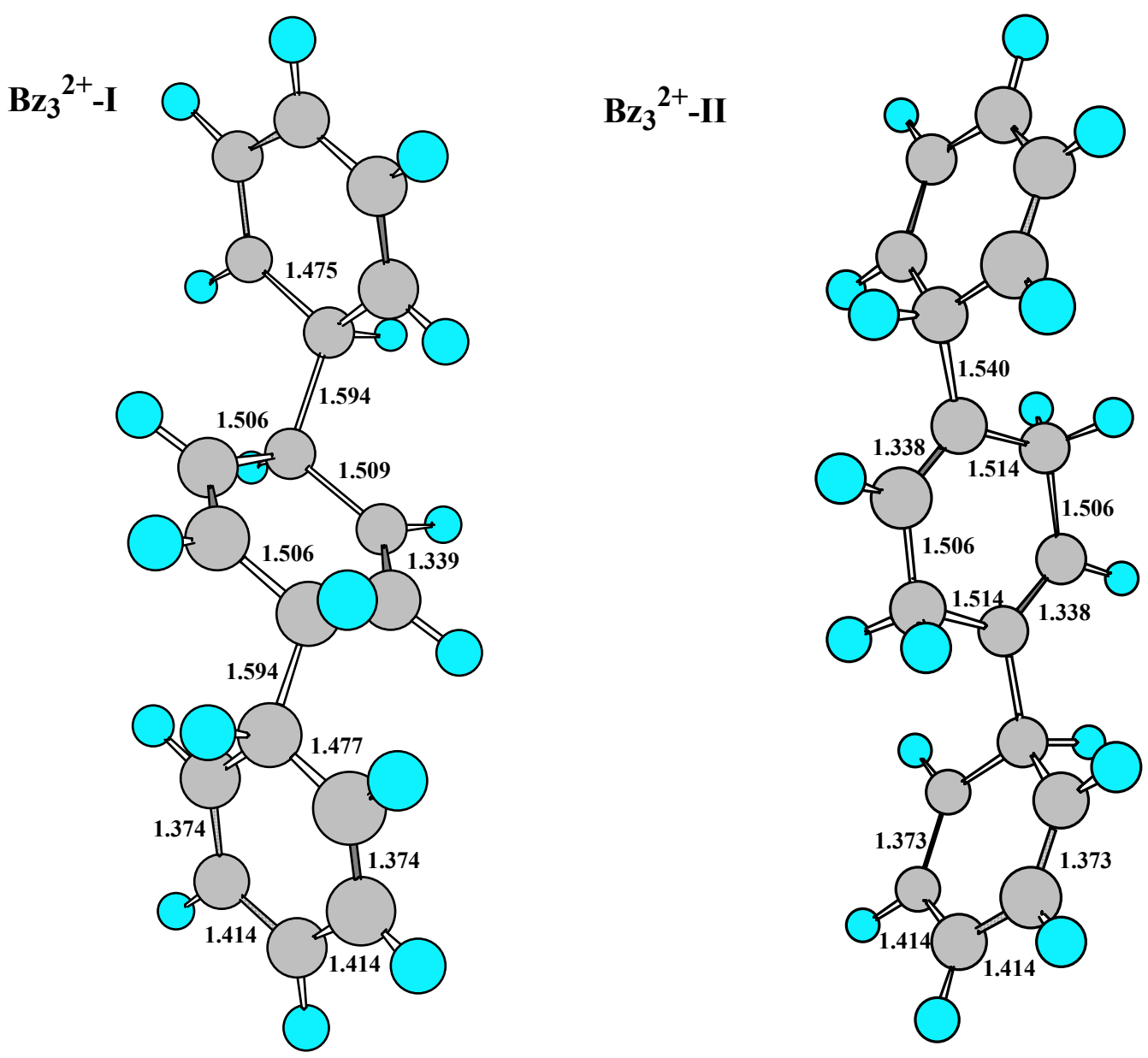

Figure 7. Identified structures of the benzene trimer dication: $\mathrm{Bz}_{3}{ }^{2+}-\mathrm{I}$ (the B3LYP/cc-pVDZ level) and $\mathrm{Bz}_{3}{ }^{2+}$-II (the B3LYP/6-31+G(d) level). Bond lengths are given in $\AA$. 
From a structural viewpoint, the mediating benzene rings in $\mathrm{Bz}_{3}{ }^{2+}-\mathrm{I}$ and $\mathrm{Bz}_{3}{ }^{2+}-\mathrm{II}$ are very peculiar, compared with the two terminal ones, which resemble those seen in $\mathrm{Bz}_{2}{ }^{2+}-\mathrm{I}$. As shown in Figure 7, these central rings contain in both trimer species two parallel $\mathrm{C}=\mathrm{C}$ double bonds $(1.34 \AA)$ that are separated by two $\mathrm{C}$ atoms bearing four substituents, among which two hydrogens. Also, the four $\mathrm{C}-\mathrm{C}$ bonds that are anchored to these carbon atoms have bond lengths $(\sim 1.51 \AA)$ that are consistent with $\sigma$ single bonds derived from $\mathrm{C}$ atoms in a $\mathrm{sp}^{3}$ hybridization state. These structural features suggest that, from a quantummechanical point of view, the two terminal rings in the dicationic trimers hold a larger part of the two electron holes. Also, in straightforward analogy with what has been reported in section 3 for $\mathrm{Bz}_{2}{ }^{2+}-\mathrm{I}$, a complex series of proton transfers around the terminal rings in $\mathrm{Bz}_{3}{ }^{2+}-\mathrm{I}$ and $\mathrm{Bz}_{3}{ }^{2+}-\mathrm{II}$ is expected to enable a further dissipation of Coulomb repulsion forces, and hence stabilization of these dicationic trimers, until the distance between the two holes is maximized.

A striking feature of the structures computed for the benzene trimer dication is the prevalence of an helix pattern in the relative orientation of rings, which reflects the development of long-range multipolar interactions. Therefore, a question that immediately comes to mind is up to what extent can these reactions induced by charge migration processes continue, and what are the largest chains or networks that can be produced this way. Clearly, the present work advocates further theoretical and more complete studies of the potential energy surfaces of larger clusters using reliable enough ab initio approaches with sufficiently large enough basis sets in order to answer these exciting questions.

Covalently bound structures that are similar to $\mathrm{Bz}_{3}{ }^{2+}-\mathrm{I}$ and $\mathrm{Bz}_{3}{ }^{2+}-\mathrm{II}$ are predicted at the $\mathrm{B} 3 \mathrm{LYP} / 6$ $31+\mathrm{G}(\mathrm{d}, \mathrm{p})$ level for the hexafluorobenzene trimer dications $\left(\mathrm{C}_{6} \mathrm{~F}_{6}\right)_{3}{ }^{2+}$-I and $\left(\mathrm{C}_{6} \mathrm{~F}_{6}\right)_{3}{ }^{2+}$-II (see Figure 8). Comparing the dihedral angles pertaining to the C-F bonds anchored to the fusion ones, one can readily conclude that $\left(\mathrm{C}_{6} \mathrm{~F}_{6}\right)_{3}{ }^{2+}$-I and $\left(\mathrm{C}_{6} \mathrm{~F}_{6}\right)_{3}{ }^{2+}$-II stem from the addition, via the charge migration mechanism, of a third hexafluorine benzene molecule to $\left(\mathrm{C}_{6} \mathrm{~F}_{6}\right)_{2}{ }^{2+}-\mathrm{I}$ and $\left(\mathrm{C}_{6} \mathrm{~F}_{6}\right)_{2}{ }^{2+}$-II, respectively. Unsurprisingly therefore, $\left(\mathrm{C}_{6} \mathrm{~F}_{6}\right)_{2}{ }^{2+}-\mathrm{I}$ is slightly more stable, by $3.2 \mathrm{kcal} / \mathrm{mol}$ without $\mathrm{ZPVE}$, than $\left(\mathrm{C}_{6} \mathrm{~F}_{6}\right)_{2}{ }^{2+}-\mathrm{II}$. 
$\left(\mathbf{C}_{6} \mathbf{F}_{6}\right)_{3}{ }^{2+}-\mathrm{I}$

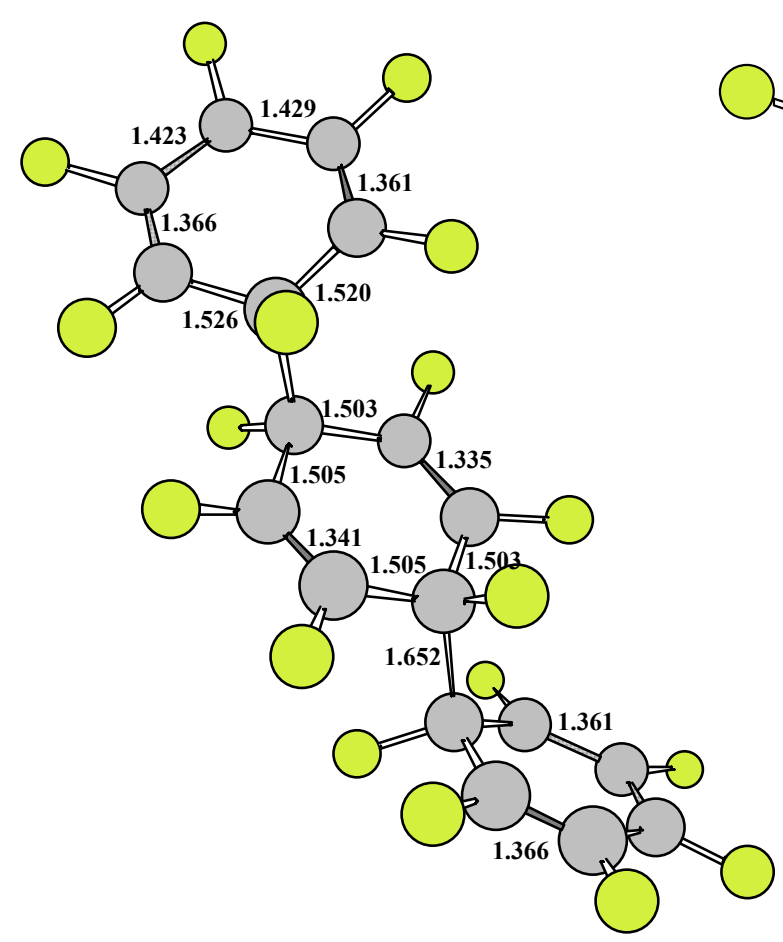

$\left(\mathrm{C}_{6} \mathrm{~F}_{6}\right)_{3}{ }^{2+}-\mathrm{II}$

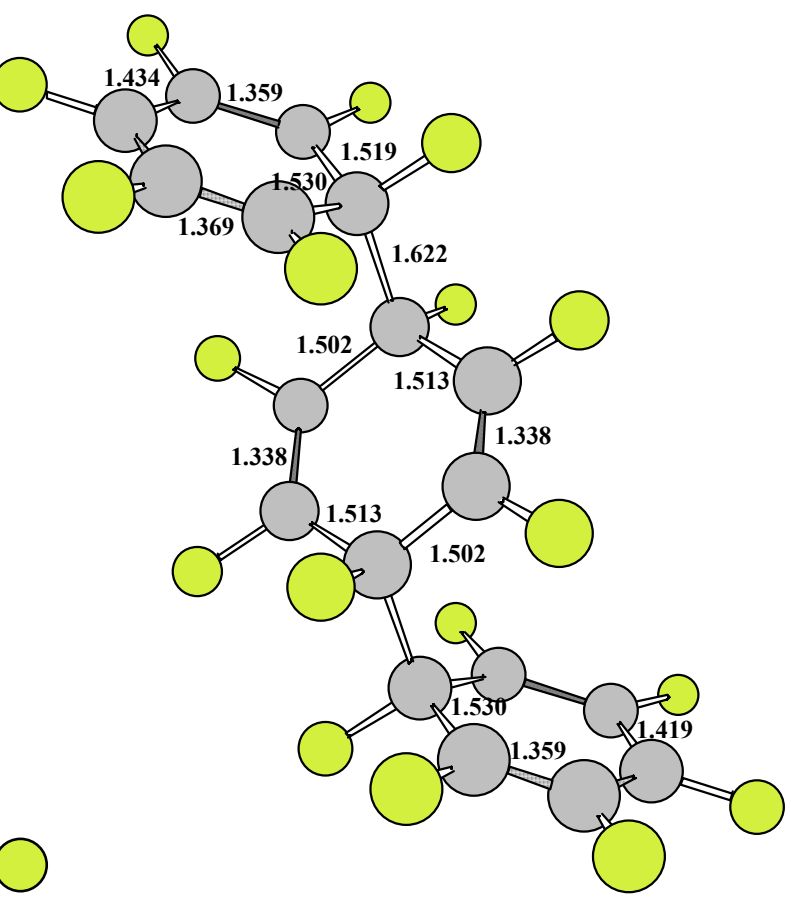

Figure 8. The identified structures of the hexafluorobenzene trimer dications $\left(\mathrm{C}_{6} \mathrm{~F}_{6}\right)_{3}{ }^{2+}-\mathrm{I}$ and $\left(\mathrm{C}_{6} \mathrm{~F}_{6}\right)_{3}{ }^{2+}$-II at the B3LYP/6-31+G(d,p) level. Bond lengths are given in $\AA$.

We focus now on some dicationic clusters containing naphthalene monomers in order to demonstrate the versatility of such covalently bound assemblies. One of the possible structures computed for the naphthalene dimer dication $\left(\mathrm{C}_{10} \mathrm{H}_{8}\right)_{2}{ }^{2+}$ is shown in Figure 9. This structure is characterized by a longer fusion bond $(1.696 \AA)$ and a slight departure, by $\sim 9^{\circ}$, from co-planarity for the rings that are immediately adjacent to the fusion bond. Two possible low-energy structures have been found at the HF/6-31G level for the naphthalene trimer dication, $\left(\mathrm{C}_{10} \mathrm{H}_{8}\right)_{3}{ }^{2+}$, and are displayed in Figure 8. The species referred to as $\left(\mathrm{C}_{10} \mathrm{H}_{8}\right)_{3}{ }^{2+}-\mathrm{II}$ is energetically more favorable, by $3.8 \mathrm{kcal} / \mathrm{mol}$ (upon accounting for ZPVE's), than the $\left(\mathrm{C}_{10} \mathrm{H}_{8}\right)_{3}{ }^{2+}-\mathrm{I}$ one. The lengths of the fusion bonds between naphthalene rings amount to 1.60 and $1.61 \AA$ in $\left(\mathrm{C}_{10} \mathrm{H}_{8}\right)_{3}{ }^{2+}-\mathrm{I}$, and to 1.58 and $1.61 \AA$ in $\left(\mathrm{C}_{10} \mathrm{H}_{8}\right)_{3}{ }^{2+}-\mathrm{II}$. It is worth mentioning that all benzenoid rings in both dicationic trimers remain planar after fusion. 


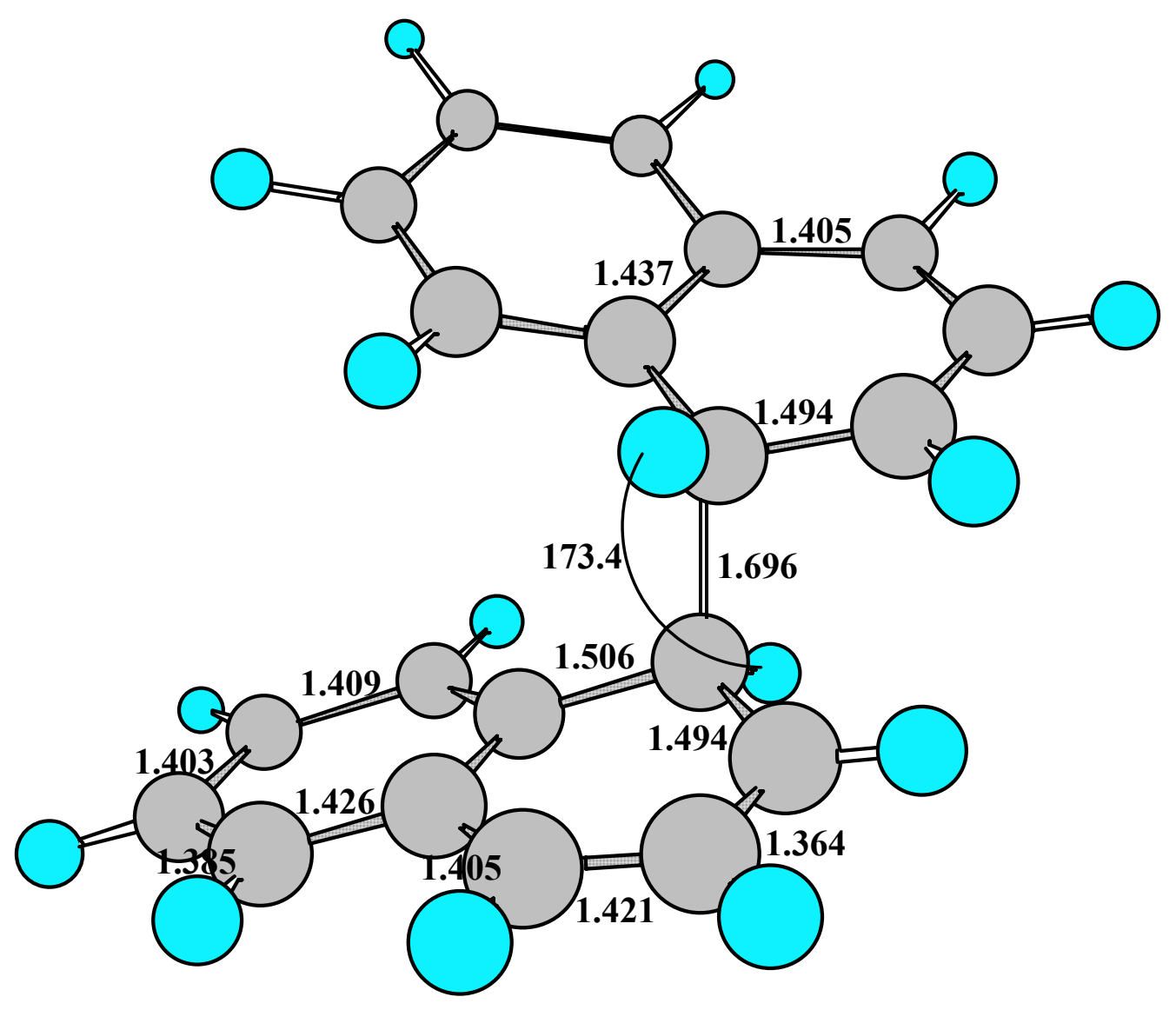

Figure 9. The structure of the naphthalene dimer dication obtained at the B3LYP/6-31+G(d) level. Bond lengths and angles are given in $\AA$ and deg, respectively.

Dicationic and hybrid assemblies of benzene and naphthalene monomers $\left[\mathrm{Bz} \cdot\left(\mathrm{C}_{10} \mathrm{H}_{8}\right) \cdot \mathrm{Bz}\right]^{2+}$ and $\left[\mathrm{Bz}_{2} \cdot\left(\mathrm{C}_{10} \mathrm{H}_{8}\right) \cdot \mathrm{Bz}_{2}\right]^{2+}$ are also shown in Figures 9 and 10 at the HF/6-31G level. Because it neglects electronic correlation, this model is known to systematically overestimate the extent of bond length alternations in extended chains, ${ }^{50}$ and thus their propensity to dissociate into non-interacting monomers units. We feel therefore confident that the stability against structural distortions and dissociation of these extremely large and hybrid assemblies of benzene and naphthalene will be confirmed with more quantitative models such as B3LYP. Both assemblies in Figures 9 and 10 are again characterized by an helix orientation pattern. The fusion bonds between the benzene and naphthalene moieties have lengths equal to $1.60 \AA$ and to $1.58 \AA$ in the $\left[\mathrm{Bz}-\left(\mathrm{C}_{10} \mathrm{H}_{8}\right)-\mathrm{Bz}\right]^{2+}$ and $\left[\mathrm{Bz}-\mathrm{Bz}-\left(\mathrm{C}_{10} \mathrm{H}_{8}\right)-\mathrm{Bz}-\mathrm{Bz}\right]^{2+}$ clusters, 
respectively. In the latter dicationic assembly, the length of the fusion bond between the benzene moieties amounts to $1.61 \AA$. In line with the idea of a propagation of active reaction sites to the extremities of polymerized clusters, this implies that the two holes in the latter species are mainly localized on the terminal benzene rings. It seems therefore that the conditions for extending the charge migration reactions to even larger assemblies are fulfilled.
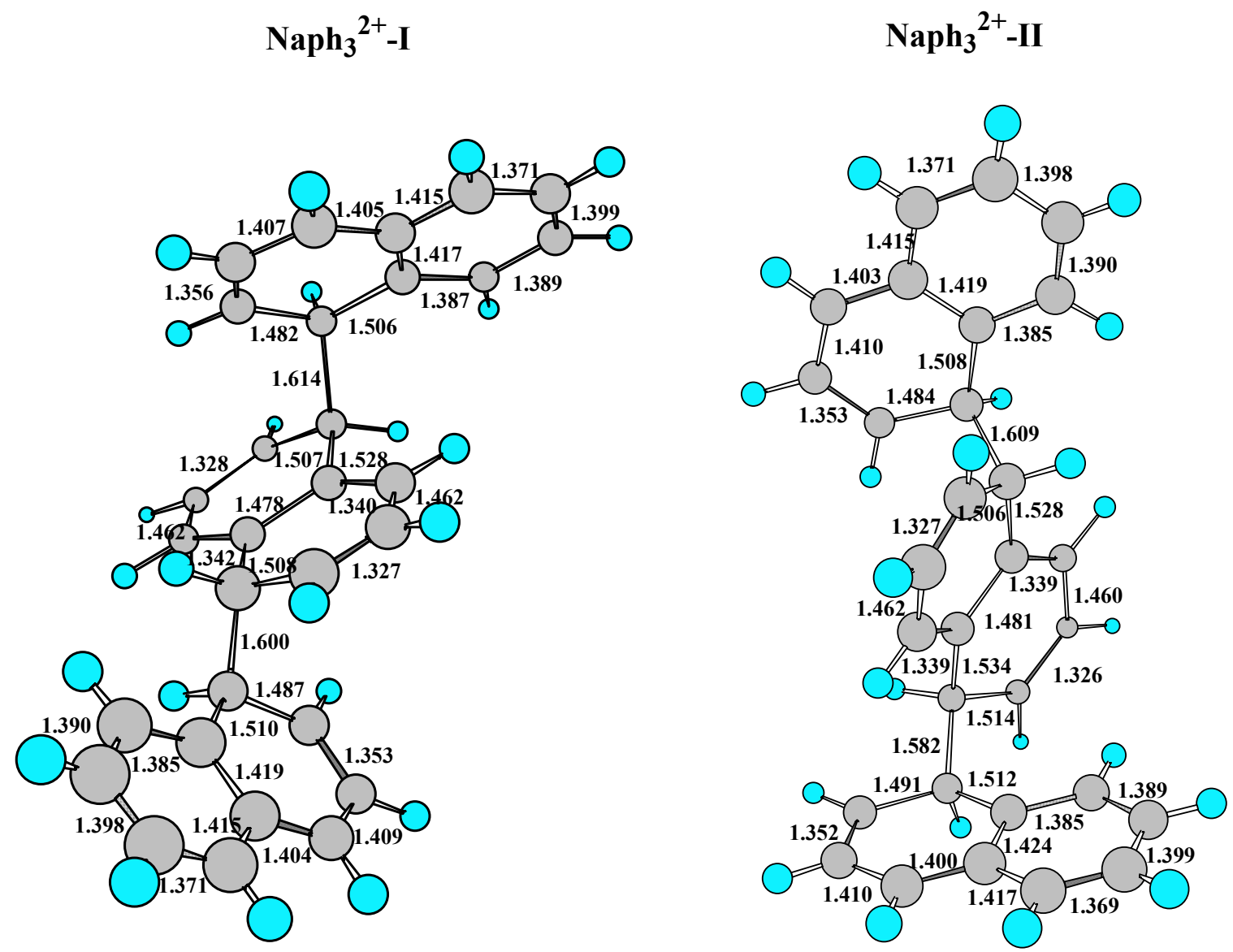

Figure 10. Two dicationic trimer assemblies of naphthalene molecules, $\mathrm{Naph}_{3}{ }^{2+}$-I and $\mathrm{Naph}_{3}{ }^{2+}$-II (the HF/6-31G level). Bond lengths are given in $\AA$. 


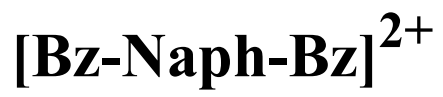

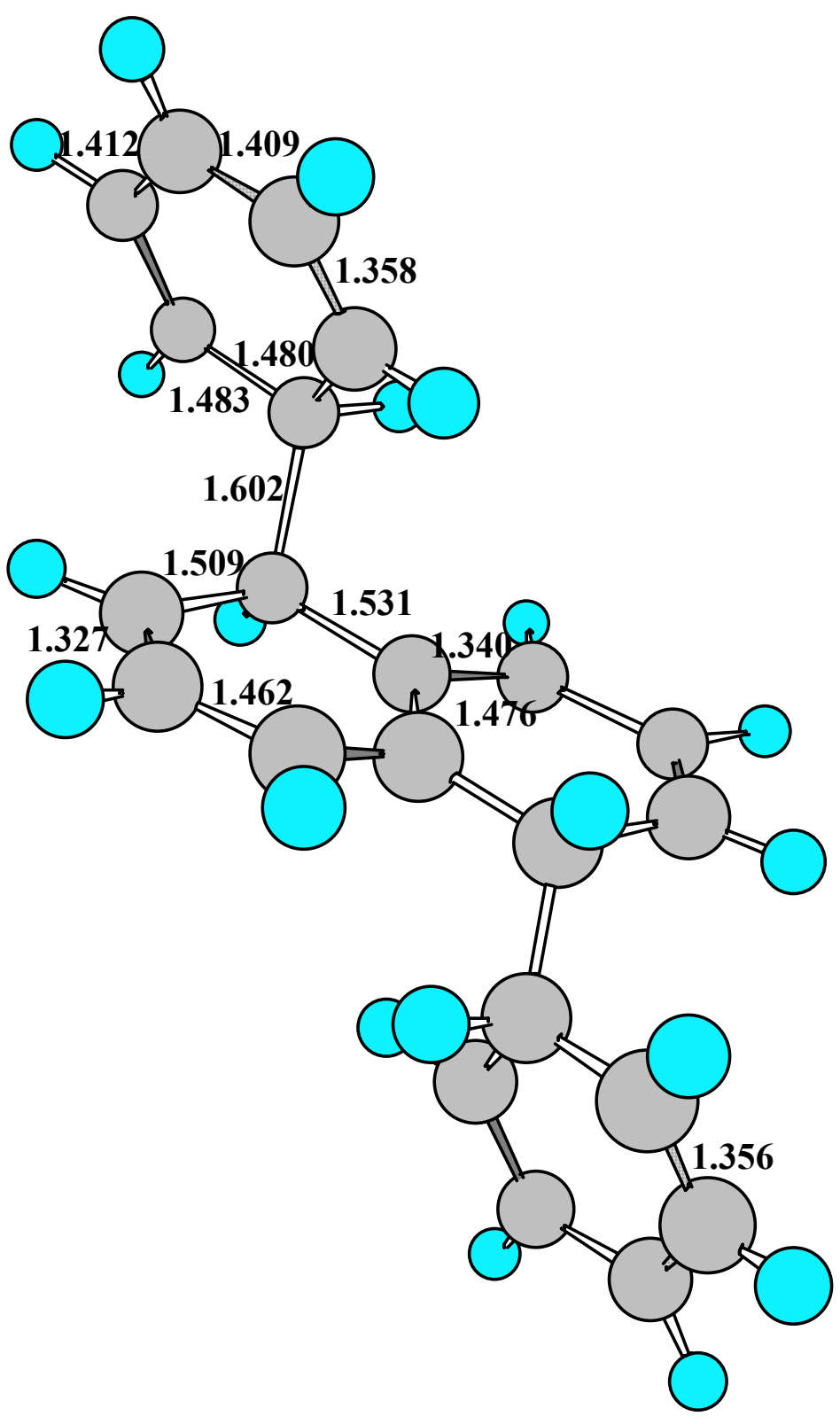

Figure 11. A hybrid dicationic trimer assembly of benzene and naphthalene molecules. The symmetric assembly of benzene-naphthalene dication $[\mathrm{Bz} \cdot \mathrm{Naph} \cdot \mathrm{Bz}]^{2+}(\mathrm{HF} / 6-31 \mathrm{G}$ level). Bond lengths are given in Å. 


\section{$\left[\mathrm{Bz}_{2}-\mathrm{Naph}-\mathrm{Bz}_{2}\right]^{2+}$}

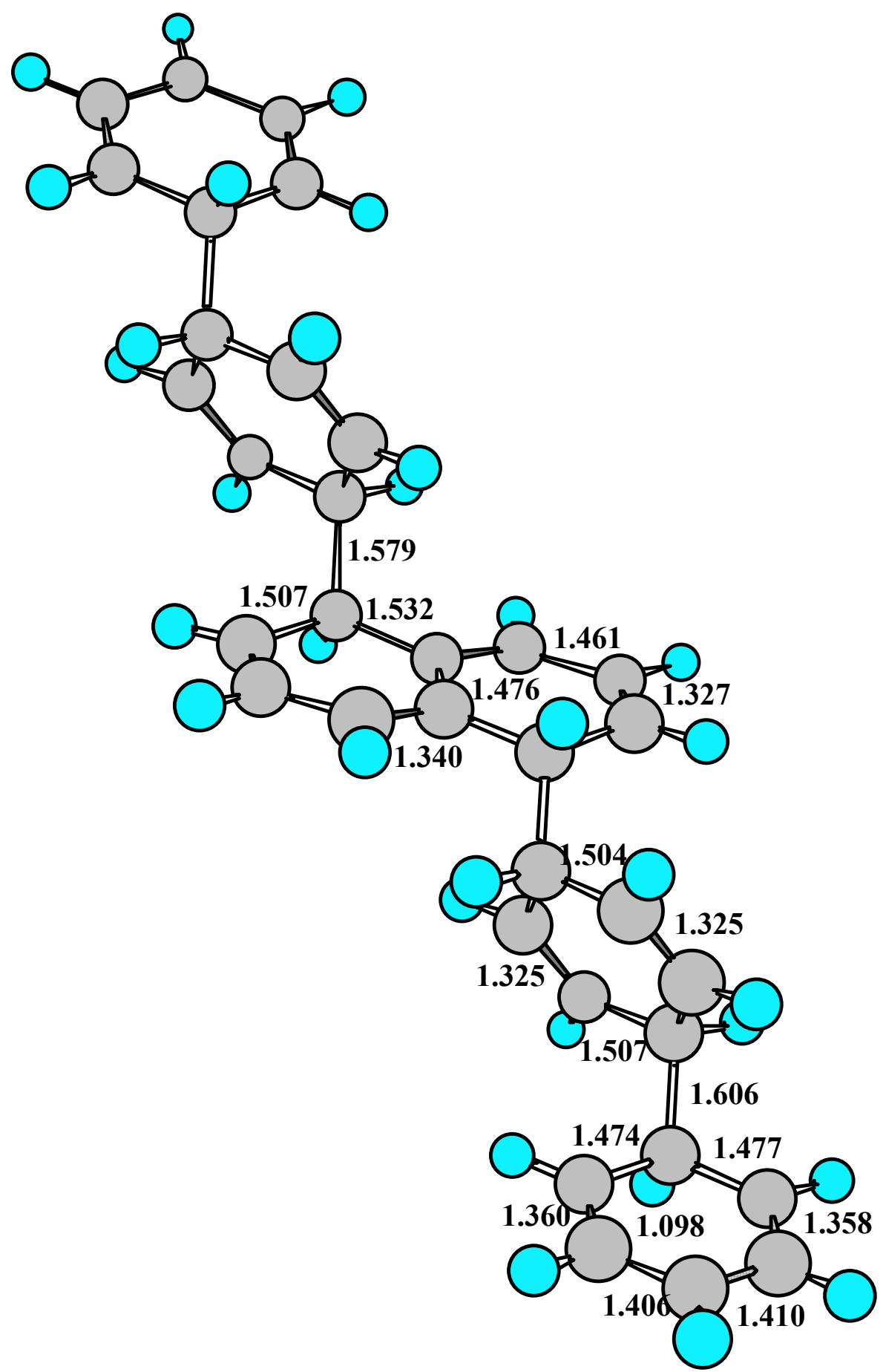

Figure 12. A hybrid dicationic pentamer assembly of benzene and naphthalene molecules $\left[\mathrm{Bz}_{2} \cdot \mathrm{Naph} \cdot \mathrm{Bz}_{2}\right]^{2+}$ (the HF/6-31G level). Bond lengths are given in $\AA$. 


\section{APPENDIX II : Optimized Geometries and Total Energies in Hartree (Atomic Units, a.u.) Obtained using Gaussian98 22}

\begin{tabular}{|c|c|c|c|c|}
\hline 24 & E (B3LYP / CC & $-\mathrm{pCDZ})=$ & -464.526503864 & a.u. \\
\hline $\mathrm{C}$ & -4.435953 & -0.232831 & -0.406955 & \\
\hline C & -3.888344 & 1.053911 & -0.429278 & \\
\hline C & -2.627530 & 1.286869 & 0.129898 & \\
\hline C & -1.913726 & 0.233195 & 0.710949 & \\
\hline C & -2.461919 & -1.053770 & 0.732607 & \\
\hline C & -3.722886 & -1.286606 & 0.174251 & \\
\hline $\mathrm{H}$ & -0.928232 & 0.413902 & 1.146474 & \\
\hline $\mathrm{H}$ & -2.200584 & 2.292690 & 0.114330 & \\
\hline $\mathrm{H}$ & -1.904182 & -1.876419 & 1.186592 & \\
\hline $\mathrm{H}$ & -4.151344 & -2.291687 & 0.191856 & \\
\hline $\mathrm{H}$ & -5.421190 & -0.414903 & -0.843109 & \\
\hline $\mathrm{H}$ & -4.445684 & 1.877149 & -0.882802 & \\
\hline C & 2.627381 & 1.286530 & -0.128939 & \\
\hline C & 3.723037 & -1.286776 & -0.175215 & \\
\hline C & 2.460476 & -1.054397 & -0.730149 & \\
\hline C & 3.889791 & 1.054034 & 0.426814 & \\
\hline $\mathrm{C}$ & 1.912125 & 0.232483 & -0.707523 & \\
\hline C & 4.437551 & -0.232629 & 0.403534 & \\
\hline $\mathrm{H}$ & 4.151617 & -2.291794 & -0.193578 & \\
\hline $\mathrm{H}$ & 5.424036 & -0.414346 & 0.837008 & \\
\hline $\mathrm{H}$ & 4.448265 & 1.877567 & 0.878406 & \\
\hline $\mathrm{H}$ & 2.200299 & 2.292282 & -0.112608 & \\
\hline $\mathrm{H}$ & 0.925375 & 0.412828 & -1.140349 & \\
\hline $\mathrm{H}$ & 1.901607 & -1.877342 & -1.182201 & \\
\hline 24 & \multicolumn{2}{|c|}{$\mathrm{E}(\mathrm{B} 3 \mathrm{LYP} / \mathrm{cC}-\mathrm{pVTZ})=$} & -464.667070919 & a.u. \\
\hline C & 4.543518 & -0.250018 & 0.410808 & \\
\hline C & 4.015730 & 1.036402 & 0.436274 & \\
\hline C & 2.766261 & 1.286252 & -0.121206 & \\
\hline C & 2.044069 & 0.249855 & -0.703581 & \\
\hline $\mathrm{C}$ & 2.572175 & -1.036727 & -0.728487 & \\
\hline $\mathrm{C}$ & 3.821853 & -1.286494 & -0.171766 & \\
\hline $\mathrm{H}$ & 1.072019 & 0.443455 & -1.137374 & \\
\hline $\mathrm{H}$ & 2.356566 & 2.287659 & -0.103375 & \\
\hline $\mathrm{H}$ & 2.010266 & -1.842525 & -1.182006 & \\
\hline $\mathrm{H}$ & 4.233054 & -2.287196 & -0.191880 & \\
\hline $\mathrm{H}$ & 5.516000 & -0.444506 & 0.843634 & \\
\hline $\mathrm{H}$ & 4.577490 & 1.842842 & 0.888981 & \\
\hline C & -2.769801 & 1.287904 & 0.120049 & \\
\hline C & -3.818308 & -1.287703 & 0.172912 & \\
\hline C & -2.570441 & -1.033492 & 0.731680 & \\
\hline C & -4.017455 & 1.033604 & -0.439478 & \\
\hline C & -2.045885 & 0.254522 & 0.705628 & \\
\hline $\mathrm{C}$ & -4.541708 & -0.254239 & -0.412848 & \\
\hline $\mathrm{H}$ & -4.226742 & -2.289518 & 0.193930 & \\
\hline $\mathrm{H}$ & -5.512784 & -0.452184 & -0.847258 & \\
\hline $\mathrm{H}$ & -4.580567 & 1.837688 & -0.894691 & \\
\hline $\mathrm{H}$ & -2.362884 & 2.290426 & 0.101315 & \\
\hline $\mathrm{H}$ & -1.075287 & 0.451586 & 1.141100 & \\
\hline $\mathrm{H}$ & -2.007181 & -1.836929 & 1.187707 & \\
\hline
\end{tabular}


Fig2b Structure of the adiabatically relaxed cation of the dimer of benzene

24

C

C

$\mathrm{C}$

C

C

C

C

C

C

$\mathrm{C}$

C

$\mathrm{H}$

$\mathrm{H}$

$\mathrm{H}$

$\mathrm{H}$

$\mathrm{H}$

$\mathrm{H}$

$\mathrm{H}$

$\mathrm{H}$

$\mathrm{H}$

$\mathrm{H}$

$\mathrm{H}$

C

C

C

C

C

C

C

C

C

C

C

C

$\mathrm{H}$

$\mathrm{H}$

$\mathrm{H}$

$\mathrm{H}$

$\mathrm{H}$

$\mathrm{H}$

$\mathrm{H}$

$\mathrm{H}$

$\mathrm{H}$

$\mathrm{H}$

$\mathrm{H}$

$\mathrm{H}$

$\mathrm{E}(\mathrm{B} 3 \mathrm{LYP} / \mathrm{cC}-\mathrm{pVDZ})=-464.224576832 \mathrm{a} \cdot \mathrm{u}$.

$$
\begin{array}{rrr}
1.976545 & 1.146827 & -0.495142 \\
1.321372 & 1.232151 & 0.769605 \\
1.131612 & 0.080283 & 1.551886 \\
1.560269 & -1.148061 & 1.069011 \\
2.208960 & -1.231496 & -0.193929 \\
2.423831 & -0.082307 & -0.966922 \\
-1.321381 & 1.232163 & -0.769583 \\
-1.976557 & 1.146813 & 0.495161 \\
-2.423833 & -0.082333 & 0.966918 \\
-2.208951 & -1.231507 & 0.193906 \\
-1.560258 & -1.148047 & -1.069030 \\
-1.131610 & 0.080309 & -1.551882 \\
0.657683 & 0.156907 & 2.531430 \\
1.430217 & -2.053467 & 1.665045 \\
1.030072 & 2.211066 & 1.156158 \\
2.144038 & 2.057305 & -1.074426 \\
2.941644 & -0.153612 & -1.924723 \\
2.557734 & -2.202584 & -0.552662 \\
-1.030088 & 2.211086 & -1.156118 \\
-2.144058 & 2.057280 & 1.074459 \\
-2.941648 & -0.153659 & 1.924717 \\
-1.557718 & -2.202604 & 0.552623 \\
-0.657678 & 0.156954 & -2.531425
\end{array}
$$

$\mathrm{E}(\mathrm{B} 3 \mathrm{LYP} / \mathrm{CC}-\mathrm{pVTZ})=-464.360596600 \mathrm{a} \cdot \mathrm{u}$.

$$
\begin{array}{r}
1.979713 \\
1.344470 \\
1.172919 \\
1.604578 \\
2.235051 \\
2.428078 \\
-1.344473 \\
-1.979718 \\
-2.428080 \\
-2.235047 \\
-1.604572 \\
-1.172917 \\
0.714861 \\
1.491073 \\
1.054185 \\
2.130993 \\
2.928735 \\
2.584432 \\
-1.054190 \\
-2.131002 \\
-2.928738 \\
-2.584426 \\
-1.491064 \\
-0.714857
\end{array}
$$

$$
\begin{array}{r}
1.142732 \\
1.220480 \\
0.072206 \\
-1.144007 \\
-1.219742 \\
-0.074406 \\
1.220484 \\
1.142727 \\
-0.074415 \\
-1.219747 \\
-1.144003 \\
0.072213 \\
0.142470 \\
-2.042500 \\
2.186779 \\
2.046494 \\
-0.139120 \\
-2.177686 \\
2.186785 \\
2.046486 \\
-0.139135 \\
-2.177693 \\
-2.042493 \\
0.142484
\end{array}
$$

$$
\begin{array}{r}
-0.504361 \\
0.762238 \\
1.539095 \\
1.052533 \\
-0.211769 \\
-0.981164 \\
-0.762231 \\
0.504366 \\
0.981163 \\
0.211762 \\
-1.052538 \\
-1.539093 \\
2.514927 \\
1.642880 \\
1.150633 \\
-1.078356 \\
-1.936507 \\
-0.572477 \\
-1.150621 \\
1.078366 \\
1.936504 \\
0.572465 \\
-1.642890 \\
-2.514924
\end{array}
$$


Fig2C : $\mathrm{TS}_{\mathrm{D}}$ structure

24

$\mathrm{E}(\mathrm{B} 3 \mathrm{LYP} / \mathrm{cC}-\mathrm{pVDZ})=-463.745558414 \mathrm{a} \cdot \mathrm{u}$.

$\begin{array}{lrrr}\mathrm{C} & 1.071506 & 1.579022 & 0.000000 \\ \mathrm{C} & -1.071506 & -1.579022 & 0.000000 \\ \mathrm{C} & 1.768673 & 1.494916 & 1.252098 \\ \mathrm{C} & -1.768673 & -1.494916 & 1.252098 \\ \mathrm{C} & 1.768673 & 1.494916 & -1.252098 \\ \mathrm{C} & -1.768673 & -1.494916 & -1.252098 \\ \mathrm{C} & 3.141216 & 1.388821 & 1.251287 \\ \mathrm{C} & -3.141216 & -1.388821 & 1.251287 \\ \mathrm{C} & 3.141216 & 1.388821 & -1.251287 \\ \mathrm{C} & -3.141216 & -1.388821 & -1.251287 \\ \mathrm{C} & 3.837500 & 1.325650 & 0.000000 \\ \mathrm{C} & -3.837500 & -1.325650 & 0.000000 \\ \mathrm{H} & 0.000000 & 1.799735 & 0.000000 \\ \mathrm{H} & 0.000000 & -1.799735 & 0.000000 \\ \mathrm{H} & 1.210250 & 1.565601 & 2.187370 \\ \mathrm{H} & -1.210250 & -1.565601 & 2.187370 \\ \mathrm{H} & 1.210250 & 1.565601 & -2.187370 \\ \mathrm{H} & -1.210250 & -1.565601 & -2.187370 \\ \mathrm{H} & 3.709648 & 1.361138 & 2.183103 \\ \mathrm{H} & -3.709648 & -1.361138 & 2.183103 \\ \mathrm{H} & 3.709648 & 1.361138 & -2.183103 \\ \mathrm{H} & -3.709648 & -1.361138 & -2.183103 \\ \mathrm{H} & 4.926838 & 1.219104 & 0.000000 \\ \mathrm{H} & -4.926838 & -1.219104 & 0.000000 \\ & & & \end{array}$

24 E (B3LYP/CC-pVTZ)

-463.879803308 a.u.

$\begin{array}{rrr}1.059760 & 1.576894 & 0.000000 \\ -1.059760 & -1.576894 & 0.000000 \\ 1.753454 & 1.494521 & 1.245694 \\ -1.753454 & -1.494521 & 1.245694 \\ 1.753454 & 1.494521 & -1.245694 \\ -1.753454 & -1.494521 & -1.245694 \\ 3.117448 & 1.397049 & 1.244741 \\ -3.117448 & -1.397049 & 1.244741 \\ 3.117448 & 1.397049 & -1.244741 \\ -3.117448 & -1.397049 & -1.244741 \\ 3.810254 & 1.337405 & 0.000000 \\ -3.810254 & -1.337405 & 0.000000 \\ 0.000000 & 1.799952 & 0.000000 \\ 0.000000 & -1.799952 & 0.000000 \\ 1.199438 & 1.562231 & 2.171258 \\ -1.199438 & -1.562231 & 2.171258 \\ 1.199438 & 1.562231 & -2.171258 \\ -1.199438 & -1.562231 & -2.171258 \\ 3.680773 & 1.373335 & 2.167185 \\ -3.680773 & -1.373335 & 2.167185 \\ 3.680773 & 1.373335 & -2.167185 \\ -3.680773 & -1.373335 & -2.167185 \\ 4.888927 & 1.235397 & 0.000000 \\ -4.888927 & -1.235397 & 0.000000\end{array}$


Fig. 2d : $\mathrm{TS}_{\mathrm{I}}$ structure

$\begin{array}{lrrr}24 & \text { E (B3LYP/CC-pVDZ })=-463.750006891 \mathrm{a} . \mathrm{u} \\ \mathrm{C} & -2.280645 & 0.013553 & 0.853188 \\ \mathrm{C} & -1.886906 & -1.188882 & 0.270366 \\ \mathrm{C} & -1.146449 & -1.205311 & -0.990301 \\ \mathrm{C} & -0.836860 & -0.013702 & -1.642407 \\ \mathrm{C} & -1.133368 & 1.191380 & -1.010746 \\ \mathrm{C} & -1.870586 & 1.202850 & 0.251528 \\ \mathrm{H} & -0.361004 & -0.024817 & -2.624613 \\ \mathrm{H} & -0.954109 & -2.171007 & -1.466791 \\ \mathrm{H} & -0.932582 & 2.147054 & -1.503574 \\ \mathrm{H} & -2.143753 & 2.168466 & 0.688241 \\ \mathrm{H} & -2.877855 & 0.024942 & 1.767062 \\ \mathrm{H} & -2.171913 & -2.144416 & 0.721297 \\ \mathrm{C} & 1.146622 & -1.205212 & 0.990556 \\ \mathrm{C} & 1.870389 & 1.202791 & -0.251784 \\ \mathrm{C} & 1.133640 & 1.191481 & 1.010761 \\ \mathrm{C} & 1.886641 & -1.188940 & -0.270361 \\ \mathrm{C} & 0.837280 & -0.013525 & 1.642647 \\ \mathrm{C} & 2.280246 & 0.013420 & -0.853425 \\ \mathrm{H} & 2.143343 & 2.168350 & -0.688747 \\ \mathrm{H} & 2.877110 & 0.024693 & -1.767525 \\ \mathrm{H} & 2.171407 & -2.144529 & -0.721322 \\ \mathrm{H} & 0.954408 & -2.170851 & 1.467216 \\ \mathrm{H} & 0.361843 & -0.024524 & 2.625060 \\ \mathrm{H} & 0.933081 & 2.147216 & 1.503568\end{array}$

$\mathrm{E}(\mathrm{B} 3 \mathrm{LYP} / \mathrm{cC}-\mathrm{pVTZ})=-463.883203668 \mathrm{a} \cdot \mathrm{u}$.

$\begin{array}{ll}\text { C } & -2.271941\end{array}$

C $\quad-1.884903$

$-1.158021$

0.012859

0.860973

$-1.181691$

$-1.197316$

0.278516

$-0.854784$

$-0.013006$

1.184084

$-0.981888$

$-1.145550$

$-1.869245$

1.194958

$-1.631344$

$-1.001343$

$-0.391595$

$-0.023587$

0.260615

$-0.969961$

$-0.949859$

$-2.153241$

$-2.607703$

2. 130595

2.150885

$-1.454220$

$-2.134486$

$-2.855292$

$-2.161900$

1.158180

0.023710

$-1.489219$

0.695768

$-2.128116$

1.770993

0.726800

$-1.197224$

0.982121

1.869067

1.194904

1.184177

$-0.260848$

1. 001348

1.884662

$-1.181743$

$-0.278508$

$-0.012841$

1. 631559

2.271577

0.012736

$-0.861185$

2. 134134

2. 150780

$-0.696214$

2.854626

0.023478

$-1.771399$

2. 161451

$-2.128221$

$-0.726806$

0.970233

$-2.153096$

1. 454607

0.392345

0.950297

$-0.023312$

2.608097

1. 489198 
Fig2e and $3: \mathrm{Bz}_{2}{ }^{2+}-\mathrm{I}$

\begin{tabular}{lrrr}
24 & \multicolumn{3}{l}{ E (B3LYP/CC-pVDZ $=-463.802503417 \mathrm{a} . \mathrm{u}}$. \\
$\mathrm{C}$ & 0.418539 & 0.663170 & 0.643835 \\
$\mathrm{C}$ & -0.418539 & -0.663170 & 0.643835 \\
$\mathrm{C}$ & 1.428447 & 0.776152 & -0.441200 \\
$\mathrm{C}$ & -1.428447 & -0.776152 & -0.441200 \\
$\mathrm{C}$ & 1.738283 & 1.990198 & -1.001668 \\
$\mathrm{C}$ & -1.738283 & -1.990198 & -1.001668 \\
$\mathrm{C}$ & 1.028550 & 3.147172 & -0.595453 \\
$\mathrm{C}$ & -1.028550 & -3.147172 & -0.595453 \\
$\mathrm{C}$ & 0.000000 & 3.105287 & 0.378459 \\
$\mathrm{C}$ & 0.000000 & -3.105287 & 0.378459 \\
$\mathrm{C}$ & -0.336063 & 1.905657 & 0.954398 \\
$\mathrm{C}$ & 0.336063 & -1.905657 & 0.954398 \\
$\mathrm{H}$ & 1.078628 & 0.598786 & 1.555148 \\
$\mathrm{H}$ & -1.078628 & -0.598786 & 1.555148 \\
$\mathrm{H}$ & 1.971869 & -0.124614 & -0.743265 \\
$\mathrm{H}$ & -1.971869 & 0.124614 & -0.743265 \\
$\mathrm{H}$ & 2.516628 & 2.070407 & -1.763383 \\
$\mathrm{H}$ & -2.516628 & -2.070407 & -1.763383 \\
$\mathrm{H}$ & 1.282758 & 4.109427 & -1.052516 \\
$\mathrm{H}$ & -1.282758 & -4.109427 & -1.052516 \\
$\mathrm{H}$ & -0.521120 & 4.023281 & 0.658074 \\
$\mathrm{H}$ & 0.521120 & -4.023281 & 0.658074 \\
$\mathrm{H}$ & -1.121698 & 1.861209 & 1.715718 \\
$\mathrm{H}$ & 1.121698 & -1.861209 & 1.715718 \\
& & &
\end{tabular}

$\mathrm{E}(\mathrm{B} 3 \mathrm{LYP} / \mathrm{cC}-\mathrm{pVTZ})=-463.932952734 \mathrm{a} \cdot \mathrm{u}$.
0.422207
0.660748
0.649616
$-0.422207$
$-0.660748$
0.649616
1. 423287
0.770094
$-0.435646$
$-1.423287$
1. 723400
$-0.770094$
$-0.435646$
1. 972909
$-1.002596$
$-1.723400$
$-1.972909$
$-1.002596$
1. 018110
3. 124935
$-3.124935$
3.087702
$-0.600784$
$-1.018110$
0.000000
$-3.087702$
$-0.600784$
0.000000
1.899671
0.373215
0.373215
$-0.327941$
0.327941
1. 074934
$-1.899671$
0.955595
0.955595
0.586803
1. 553159
1. 553159
-1.062507
1.962507
$-1.962507$
$-0.586803$
$-0.729948$
0.122519
$-0.729948$
2. 490041
$-2.490041$
1. 266932
2. 050287
$-1.761347$
$-2.050287$
$-1.761347$
4.075474
$-1.059223$
$-1.266932$
$-4.075474$
$-1.059223$
$-0.515467$
0.515467
$-1.101171$
- 998182
0.646736
0.646736
1. 714225
1. 101171
1. 857845
1.714225 
Fig $2 \mathrm{f}$ and $3: \mathrm{Bz}_{2}{ }^{2+}$-Its

$\begin{array}{lrrr}24 & \text { E (B3LYP/CC-PVDZ }=-463.796903723 \mathrm{a} . \mathrm{u} . \\ \mathrm{C} & 0.000000 & 0.794767 & 0.000000 \\ \mathrm{C} & 0.000000 & -0.794767 & 0.000000 \\ \mathrm{C} & 0.471252 & 1.444668 & 1.252933 \\ \mathrm{C} & -0.471252 & -1.444668 & 1.252933 \\ \mathrm{C} & 0.471252 & 1.444668 & -1.252933 \\ \mathrm{C} & -0.471252 & -1.444668 & -1.252933 \\ \mathrm{C} & 1.180778 & 2.619170 & 1.237385 \\ \mathrm{C} & -1.180778 & -2.619170 & 1.237385 \\ \mathrm{C} & 1.180778 & 2.619170 & -1.237385 \\ \mathrm{C} & -1.180778 & -2.619170 & -1.237385 \\ \mathrm{C} & 1.546360 & 3.200902 & 0.000000 \\ \mathrm{C} & -1.546360 & -3.200902 & 0.000000 \\ \mathrm{H} & -1.088246 & 1.089368 & 0.000000 \\ \mathrm{H} & 1.088246 & -1.089368 & 0.000000 \\ \mathrm{H} & 0.181962 & 1.018106 & 2.215421 \\ \mathrm{H} & -0.181962 & -1.018106 & 2.215421 \\ \mathrm{H} & 0.181962 & 1.018106 & -2.215421 \\ \mathrm{H} & -0.181962 & -1.018106 & -2.215421 \\ \mathrm{H} & 1.471848 & 3.102212 & 2.172357 \\ \mathrm{H} & -1.471848 & -3.102212 & 2.172357 \\ \mathrm{H} & 1.471848 & 3.102212 & -2.172357 \\ \mathrm{H} & -1.471848 & -3.102212 & -2.172357 \\ \mathrm{H} & 2.125839 & 4.130247 & 0.000000 \\ \mathrm{H} & -2.125839 & -4.130247 & 0.000000\end{array}$

$E(B 3 L Y P / c c-p V T Z)=-463.927710577 \mathrm{a} \cdot \mathrm{u}$.
0.000000
0.795736
0.000000
0.000000
$-0.795736$
0.000000
0.480384
1. 436184
1.247474
$-0.480384$
$-1.436184$
1. 247474
0.480384
1. 436184
$-1.247474$
$-0.480384$
$-1.436184$
$-1.247474$
2. 587770
1. 231107
$-1.208912$
$-2.587770$
1.231107
1.208912
2.587770
$-1.231107$
$-1.208912$
$-2.587770$
$-1.231107$
3.158885
0.000000
$-1.583313$
$-3.158885$
0.000000
$-1.078911$
1.083095
$-1.083095$
0.000000
0.000000
2.199387
0.184119
1.018975
2.199387
$-0.184119$
$-1.018975$
1. 018975
$-2.199387$
$-0.184119$
$-1.018975$
3. 061157
$-2.199387$
2.156360
2.156360
$-1.507531$
$-3.061157$
3.061157
$-2.156360$
$-1.507531$
2.174505
$-3.061157$
$-2.156360$
4. 067714
0.000000
$-2.174505$
$-4.067714$
0.000000 
Fig3 : $\mathrm{Bz}_{2}{ }^{2+}-$ IIts

$24 \mathrm{E}(\mathrm{B} 3 \mathrm{LYP} / \mathrm{CC}-\mathrm{pVDZ})=-463.786791787 \mathrm{a} \cdot \mathrm{u}$.

$\begin{array}{lrrr}\text { C } & 1.382113 & -1.117765 & -0.629160 \\ \mathrm{C} & 0.771246 & -0.355565 & 0.499315 \\ \mathrm{C} & 1.592157 & 0.762520 & 1.043727 \\ \mathrm{C} & 2.886155 & 0.956856 & 0.633579 \\ \mathrm{C} & 3.424673 & 0.138194 & -0.391817 \\ \mathrm{C} & 2.678094 & -0.890780 & -1.017735 \\ \mathrm{C} & -0.723841 & -0.069092 & 0.289111 \\ \mathrm{C} & -1.734647 & -1.040501 & 0.712404 \\ \mathrm{C} & -3.093757 & -0.843736 & 0.368071 \\ \mathrm{C} & -3.462016 & 0.259779 & -0.391252 \\ \mathrm{C} & -2.494153 & 1.201131 & -0.813685 \\ \mathrm{C} & -1.154086 & 1.046975 & -0.491238 \\ \mathrm{H} & -0.418571 & 1.776477 & -0.837242 \\ \mathrm{H} & -2.806173 & 2.062616 & -1.409207 \\ \mathrm{H} & -1.334076 & 0.021055 & 1.434944 \\ \mathrm{H} & -1.424288 & -1.918265 & 1.286721 \\ \mathrm{H} & -3.833689 & -1.573617 & 0.703200 \\ \mathrm{H} & -4.510324 & 0.405615 & -0.663192 \\ \mathrm{H} & 0.854446 & -1.107807 & 1.332290 \\ \mathrm{H} & 1.162180 & 1.385156 & 1.834579 \\ \mathrm{H} & 3.500425 & 1.741855 & 1.079700 \\ \mathrm{H} & 4.456480 & 0.311568 & -0.715924 \\ \mathrm{H} & 3.133128 & -1.486389 & -1.812011 \\ \mathrm{H} & 0.788838 & -1.906352 & -1.101775\end{array}$

$24 \mathrm{E}(\mathrm{B} 3 \mathrm{LYP} / \mathrm{CC}-\mathrm{pVDZ})=-463.917203883 \mathrm{a} \cdot \mathrm{u}$.

$\begin{array}{lrrr}\mathrm{C} & 1.380038 & -1.122696 & -0.611233 \\ \mathrm{C} & 0.769471 & -0.349718 & 0.502295 \\ \mathrm{C} & 1.586891 & 0.771643 & 1.028688 \\ \mathrm{C} & 2.870851 & 0.961975 & 0.616137 \\ \mathrm{C} & 3.406876 & 0.134838 & -0.393040 \\ \mathrm{C} & 2.665763 & -0.897847 & -1.002060 \\ \mathrm{C} & -0.722047 & -0.065915 & 0.287166 \\ \mathrm{C} & -1.725778 & -1.035141 & 0.706369 \\ \mathrm{C} & -3.077310 & -0.843407 & 0.362566 \\ \mathrm{C} & -3.445057 & 0.254600 & -0.389383 \\ \mathrm{C} & -2.484811 & 1.194349 & -0.808071 \\ \mathrm{C} & -1.152742 & 1.044061 & -0.488098 \\ \mathrm{H} & -0.425952 & 1.769030 & -0.829118 \\ \mathrm{H} & -2.796816 & 2.047490 & -1.396034 \\ \mathrm{H} & -1.327744 & 0.022480 & 1.422505 \\ \mathrm{H} & -1.415097 & -1.903652 & 1.274302 \\ \mathrm{H} & -3.808933 & -1.568149 & 0.692399 \\ \mathrm{H} & -4.483248 & 0.397755 & -0.658629 \\ \mathrm{H} & 0.846178 & -1.083346 & 1.340189 \\ \mathrm{H} & 1.160141 & 1.396483 & 1.804437 \\ \mathrm{H} & 3.478911 & 1.745964 & 1.046447 \\ \mathrm{H} & 4.428187 & 0.303244 & -0.716231 \\ \mathrm{H} & 3.117776 & -1.496370 & -1.781210 \\ \mathrm{H} & 0.793725 & -1.911385 & -1.067080\end{array}$




\begin{tabular}{lrrr} 
Fig3 $: \mathrm{Bz}_{2}{ }^{2+}-\mathrm{II}$ & \\
$\mathrm{H}$ & & \\
& $\mathrm{H}(\mathrm{B} 3 \mathrm{LYP} / \mathrm{CC}-\mathrm{pVDZ})$ & \multicolumn{3}{l}{$-463.812831409 \mathrm{a} \cdot \mathrm{u}}$. \\
$\mathrm{C}$ & -1.665210 & -1.230174 & 0.374503 \\
$\mathrm{C}$ & -0.728707 & -0.265831 & 0.099202 \\
$\mathrm{C}$ & -1.179982 & 1.090378 & -0.309596 \\
$\mathrm{C}$ & -2.632641 & 1.323237 & -0.402075 \\
$\mathrm{C}$ & -3.542262 & 0.328199 & -0.118607 \\
$\mathrm{C}$ & -3.055084 & -0.934506 & 0.266257 \\
$\mathrm{C}$ & 0.770817 & -0.552930 & 0.195191 \\
$\mathrm{C}$ & 1.504448 & 0.159631 & 1.278705 \\
$\mathrm{C}$ & 2.813458 & 0.540482 & 1.118504 \\
$\mathrm{C}$ & 3.455286 & 0.329972 & -0.126857 \\
$\mathrm{C}$ & 2.799424 & -0.272145 & -1.228753 \\
$\mathrm{C}$ & 1.490233 & -0.666188 & -1.105365 \\
$\mathrm{H}$ & -0.752160 & 1.867327 & 0.365726 \\
$\mathrm{H}$ & -2.967949 & 2.320786 & -0.703378 \\
$\mathrm{H}$ & -0.719182 & 1.385343 & -1.280365 \\
$\mathrm{H}$ & -1.373080 & -2.237620 & 0.679979 \\
$\mathrm{H}$ & -3.770334 & -1.731607 & 0.494826 \\
$\mathrm{H}$ & -4.616981 & 0.506423 & -0.187502 \\
$\mathrm{H}$ & 0.860599 & -1.615215 & 0.562311 \\
$\mathrm{H}$ & 0.995720 & 0.304279 & 2.236786 \\
$\mathrm{H}$ & 3.360818 & 1.009240 & 1.939111 \\
$\mathrm{H}$ & 4.498168 & 0.643868 & -0.241836 \\
$\mathrm{H}$ & 3.335840 & -0.412289 & -2.169709 \\
$\mathrm{H}$ & 0.969856 & -1.141285 & -1.942608
\end{tabular}

$-1.176606$

0.768710

$-0.555294$

0.252633

1.500338

0.136945

0.185673

2.799523

0.520860

1.275575

3.437764

0.332416

1.121177

2.786296

1.486916

$-0.249140$

$-0.646058$

1. 850281

$-0.751907$

2. 312718

1.394676

$-0.721344$

$-1.367202$

$-2.229611$

$-1.725657$

$-3.747440$

0.503641

0.854383

0.996534

$-1.615037$

$-0.120754$

$-1.226387$

$-1.110525$

0.385659

$-0.665087$

$-1.253176$

0.643436

0.468662

$-0.176593$

0.532643

0.263403

2. 226411

3. 341667

0.972565

0.645773

1. 940681

4.469981

3.318269

$-0.371425$

0.972249

$-1.103921$

$-0.229430$

$-2.160010$

$-1.946708$ 
Fig3 : $\mathrm{Bz}_{2}{ }^{2+}-$ III

\begin{tabular}{lrrr}
24 & $E(B 3 L Y P / C C-P V D Z)$ & \multicolumn{3}{c}{$-463.798639452 \mathrm{a} . \mathrm{u}}$. \\
$\mathrm{C}$ & -1.508868 & -1.138908 & 0.502771 \\
$\mathrm{C}$ & -0.754069 & -0.046665 & -0.136590 \\
$\mathrm{C}$ & -1.498948 & 1.084293 & -0.609216 \\
$\mathrm{C}$ & -2.879173 & 1.116125 & -0.492787 \\
$\mathrm{C}$ & -3.587124 & 0.053131 & 0.114251 \\
$\mathrm{C}$ & -2.914103 & -1.063811 & 0.599673 \\
$\mathrm{C}$ & 0.743993 & -0.060404 & -0.098826 \\
$\mathrm{C}$ & 1.492823 & -1.119945 & -0.555165 \\
$\mathrm{C}$ & 2.912569 & -1.086812 & -0.468050 \\
$\mathrm{C}$ & 3.622130 & -0.000505 & 0.076150 \\
$\mathrm{C}$ & 2.907907 & 1.079886 & 0.545905 \\
$\mathrm{C}$ & 1.436212 & 1.114753 & 0.495309 \\
$\mathrm{H}$ & 1.123329 & 2.062434 & 0.001839 \\
$\mathrm{H}$ & 3.418591 & 1.946717 & 0.977178 \\
$\mathrm{H}$ & 1.062877 & 1.291505 & 1.531799 \\
$\mathrm{H}$ & 1.029121 & -1.996266 & -1.014920 \\
$\mathrm{H}$ & 3.471323 & -1.947060 & -0.850847 \\
$\mathrm{H}$ & 4.712720 & -0.020552 & 0.117914 \\
$\mathrm{H}$ & -1.170062 & -1.047118 & -0.818851 \\
$\mathrm{H}$ & -0.974527 & 1.910788 & -1.092637 \\
$\mathrm{H}$ & -3.428874 & 1.978677 & -0.877020 \\
$\mathrm{H}$ & -4.675148 & 0.109672 & 0.200143 \\
$\mathrm{H}$ & -3.452639 & -1.884268 & 1.078871 \\
$\mathrm{H}$ & -0.956807 & -1.991359 & 0.905973
\end{tabular}

$24 \mathrm{E}(\mathrm{B} 3 \mathrm{LYP} / \mathrm{CC}-\mathrm{PVTZ})=$

$=-463.929627114 \mathrm{a} \cdot \mathrm{u}$.

$\begin{array}{lrrr}\mathrm{C} & -1.504037 & -1.135060 & 0.495936 \\ \mathrm{C} & -0.751530 & -0.046466 & -0.135954 \\ \mathrm{C} & -1.492615 & 1.081682 & -0.600072 \\ \mathrm{C} & -2.864083 & 1.112577 & -0.485989 \\ \mathrm{C} & -3.568346 & 0.052463 & 0.113092 \\ \mathrm{C} & -2.900591 & -1.059548 & 0.593262 \\ \mathrm{C} & 0.740871 & -0.061068 & -0.097497 \\ \mathrm{C} & 1.484171 & -1.114944 & -0.547697 \\ \mathrm{C} & 2.896330 & -1.083523 & -0.461996 \\ \mathrm{C} & 3.603785 & -0.002386 & 0.075434 \\ \mathrm{C} & 2.897479 & 1.073806 & 0.539363 \\ \mathrm{C} & 1.431637 & 1.111901 & 0.488774 \\ \mathrm{H} & 1.124675 & 2.046980 & -0.010661 \\ \mathrm{H} & 3.403680 & 1.932908 & 0.963481 \\ \mathrm{H} & 1.059247 & 1.297231 & 1.513389 \\ \mathrm{H} & 1.023016 & -1.983920 & -0.999436 \\ \mathrm{H} & 3.448653 & -1.937116 & -0.838339 \\ \mathrm{H} & 4.683796 & -0.024498 & 0.116130 \\ \mathrm{H} & -1.162488 & -1.032775 & -0.818604 \\ \mathrm{H} & -0.972080 & 1.902106 & -1.074186 \\ \mathrm{H} & -3.409017 & 1.967898 & -0.862975 \\ \mathrm{H} & -4.645779 & 0.108467 & 0.196867 \\ \mathrm{H} & -3.435419 & -1.872948 & 1.064354 \\ \mathrm{H} & -0.956712 & -1.980939 & 0.890038\end{array}$


Fig3 : $\mathrm{Bz}_{2}{ }^{2+}-\mathrm{III}$

$24 \mathrm{E}(\mathrm{B} 3 \mathrm{LYP} / \mathrm{CC}-\mathrm{pVDZ})=-463.829966036 \mathrm{a} \cdot \mathrm{u}$.

$\begin{array}{lrrr}\mathrm{C} & -1.531732 & 1.243348 & 0.000497 \\ \mathrm{C} & -0.738506 & -0.021759 & -0.000110 \\ \mathrm{C} & -1.433446 & -1.218599 & -0.000687 \\ \mathrm{C} & -2.854277 & -1.247978 & -0.000597 \\ \mathrm{C} & -3.643713 & -0.084858 & 0.000159 \\ \mathrm{C} & -3.001078 & 1.135194 & 0.000793 \\ \mathrm{C} & 0.738509 & 0.021778 & -0.000131 \\ \mathrm{C} & 1.531718 & -1.243342 & 0.000427 \\ \mathrm{C} & 3.001064 & -1.135204 & 0.000856 \\ \mathrm{C} & 3.643711 & 0.084840 & 0.000206 \\ \mathrm{C} & 2.854290 & 1.247969 & -0.000619 \\ \mathrm{C} & 1.433460 & 1.218609 & -0.000746 \\ \mathrm{H} & -1.251710 & 1.887888 & 0.863420 \\ \mathrm{H} & -3.572802 & 2.068666 & 0.001472 \\ \mathrm{H} & -1.252390 & 1.888026 & -0.862578 \\ \mathrm{H} & -0.919317 & -2.179865 & -0.001281 \\ \mathrm{H} & -3.347866 & -2.224947 & -0.001126 \\ \mathrm{H} & -4.733021 & -0.153946 & 0.000279 \\ \mathrm{H} & 1.252565 & -1.887709 & -0.862967 \\ \mathrm{H} & 0.919346 & 2.179882 & -0.001375 \\ \mathrm{H} & 3.347891 & 2.224932 & -0.001143 \\ \mathrm{H} & 4.733020 & 0.153918 & 0.000388 \\ \mathrm{H} & 3.572774 & -2.068685 & 0.001576 \\ \mathrm{H} & 1.251505 & -1.888150 & 0.863055\end{array}$

$24 \mathrm{E}(\mathrm{B} 3 \mathrm{LYP} / \mathrm{cC}-\mathrm{pVTZ})=-463.960586081 \mathrm{a} \cdot \mathrm{u}$.

$\begin{array}{lrrr}\mathrm{C} & -1.527564 & 1.238137 & 0.000524 \\ \mathrm{C} & -0.735672 & -0.021843 & -0.000069 \\ \mathrm{C} & -1.426116 & -1.210833 & -0.000632 \\ \mathrm{C} & -2.839329 & -1.241880 & -0.000585 \\ \mathrm{C} & -3.625996 & -0.086456 & 0.000091 \\ \mathrm{C} & -2.990648 & 1.127082 & 0.000699 \\ \mathrm{C} & 0.735673 & 0.021847 & -0.000081 \\ \mathrm{C} & 1.527561 & -1.238136 & 0.000468 \\ \mathrm{C} & 2.990645 & -1.127084 & 0.000749 \\ \mathrm{C} & 3.625996 & 0.086453 & 0.000126 \\ \mathrm{C} & 2.839331 & 1.241879 & -0.000603 \\ \mathrm{C} & 1.426119 & 1.210835 & -0.000670 \\ \mathrm{H} & -1.250717 & 1.876987 & 0.856316 \\ \mathrm{H} & -3.557543 & 2.050631 & 0.001274 \\ \mathrm{H} & -1.251222 & 1.877242 & -0.855285 \\ \mathrm{H} & -0.914777 & -2.161938 & -0.001178 \\ \mathrm{H} & -3.327172 & -2.209817 & -0.001086 \\ \mathrm{H} & -4.704621 & -0.157081 & 0.000157 \\ \mathrm{H} & 1.251358 & -1.877018 & -0.855571 \\ \mathrm{H} & 0.914783 & 2.161942 & -0.001236 \\ \mathrm{H} & 3.327177 & 2.209814 & -0.001120 \\ \mathrm{H} & 4.704621 & 0.157075 & 0.000229 \\ \mathrm{H} & 3.557538 & -2.050634 & 0.001363 \\ \mathrm{H} & 1.250572 & -1.877201 & 0.856035\end{array}$


Fig3 : $\mathrm{Bz}_{2}{ }^{2+}-\mathrm{IV}$

$24 \mathrm{E}(\mathrm{B} 3 \mathrm{LYP} / \mathrm{cC}-\mathrm{pVDZ})=-463.829149265 \mathrm{a} \cdot \mathrm{u}$.

$\begin{array}{lrrr}\mathrm{C} & -1.452776 & 1.201555 & -0.315903 \\ \mathrm{C} & -0.738604 & 0.059000 & -0.001880 \\ \mathrm{C} & -1.497496 & -1.183611 & 0.323864 \\ \mathrm{C} & -2.969852 & -1.111773 & 0.301183 \\ \mathrm{C} & -3.635208 & 0.051911 & -0.017209 \\ \mathrm{C} & -2.872853 & 1.194953 & -0.323331 \\ \mathrm{C} & 0.738604 & 0.059000 & 0.001883 \\ \mathrm{C} & 1.497494 & -1.183614 & -0.323852 \\ \mathrm{C} & 2.969850 & -1.111771 & -0.301201 \\ \mathrm{C} & 3.635208 & 0.051912 & 0.017194 \\ \mathrm{C} & 2.872854 & 1.194951 & 0.323329 \\ \mathrm{C} & 1.452778 & 1.201553 & 0.315909 \\ \mathrm{H} & -1.198038 & -1.567187 & 1.326273 \\ \mathrm{H} & -3.522771 & -2.022082 & 0.553470 \\ \mathrm{H} & -4.725813 & 0.094927 & -0.033369 \\ \mathrm{H} & -0.945793 & 2.124842 & -0.599921 \\ \mathrm{H} & -3.390303 & 2.123882 & -0.583641 \\ \mathrm{H} & -1.193552 & -2.026562 & -0.336160 \\ \mathrm{H} & 0.945796 & 2.124838 & 0.599934 \\ \mathrm{H} & 1.193586 & -2.026529 & 0.336240 \\ \mathrm{H} & 3.522768 & -2.022078 & -0.553498 \\ \mathrm{H} & 4.725813 & 0.094928 & 0.033344 \\ \mathrm{H} & 3.390306 & 2.123878 & 0.583642 \\ \mathrm{H} & 1.197998 & -1.567253 & -1.326223\end{array}$

$24 \mathrm{E}(\mathrm{B} 3 \mathrm{LYP} / \mathrm{cc}-\mathrm{pVTZ})$

$=-463.960147721 \mathrm{a} \cdot \mathrm{u}$.

$\begin{array}{lrrr}\mathrm{C} & -1.443724 & 1.188490 & -0.332352 \\ \mathrm{C} & -0.735721 & 0.058437 & -0.001436 \\ \mathrm{C} & -1.492772 & -1.173937 & 0.342119 \\ \mathrm{C} & -2.958937 & -1.099462 & 0.316950 \\ \mathrm{C} & -3.616209 & 0.052869 & -0.019188 \\ \mathrm{C} & -2.856205 & 1.183686 & -0.340963 \\ \mathrm{C} & 0.735721 & 0.058437 & 0.001440 \\ \mathrm{C} & 1.492771 & -1.173939 & -0.342113 \\ \mathrm{C} & 2.958936 & -1.099461 & -0.316958 \\ \mathrm{C} & 3.616209 & 0.052869 & 0.019179 \\ \mathrm{C} & 2.856207 & 1.183685 & 0.340961 \\ \mathrm{C} & 1.443725 & 1.188488 & 0.332356 \\ \mathrm{H} & -1.194826 & -1.540630 & 1.340437 \\ \mathrm{H} & -3.507221 & -1.995936 & 0.581334 \\ \mathrm{H} & -4.696186 & 0.097681 & -0.037174 \\ \mathrm{H} & -0.938481 & 2.097027 & -0.627668 \\ \mathrm{H} & -3.367520 & 2.099881 & -0.613457 \\ \mathrm{H} & -1.190639 & -2.018492 & -0.300345 \\ \mathrm{H} & 0.938483 & 2.097025 & 0.627674 \\ \mathrm{H} & 1.190655 & -2.018479 & 0.300381 \\ \mathrm{H} & 3.507219 & -1.995934 & -0.581348 \\ \mathrm{H} & 4.696186 & 0.097682 & 0.037158 \\ \mathrm{H} & 3.367522 & 2.099879 & 0.613457 \\ \mathrm{H} & 1.194807 & -1.540659 & -1.340414 \\ & & & \end{array}$


Fig3 : $\mathrm{Bz}_{2}{ }^{2+}-\mathrm{V}$

$24 \mathrm{E}(\mathrm{B} 3 \mathrm{LYP} / \mathrm{cC}-\mathrm{pVDZ})=-463.830169819 \mathrm{a} \cdot \mathrm{u}$.

$\begin{array}{lrrr}\text { C } & 1.477707 & 1.089807 & 0.521644 \\ \mathrm{C} & 0.750098 & -0.021196 & -0.004111 \\ \mathrm{C} & 1.476765 & -1.078414 & -0.512938 \\ \mathrm{C} & 2.945907 & -1.069503 & -0.510959 \\ \mathrm{C} & 3.618311 & 0.105157 & 0.049748 \\ \mathrm{C} & 2.882122 & 1.158856 & 0.553083 \\ \mathrm{C} & -0.750099 & -0.021204 & 0.004086 \\ \mathrm{C} & -1.476758 & -1.078422 & 0.512923 \\ \mathrm{C} & -2.945899 & -1.069507 & 0.510970 \\ \mathrm{C} & -3.618312 & 0.105155 & -0.049723 \\ \mathrm{C} & -2.882130 & 1.158856 & -0.553064 \\ \mathrm{C} & -1.477715 & 1.089786 & -0.521688 \\ \mathrm{H} & 0.977551 & -1.949184 & -0.948031 \\ \mathrm{H} & 3.330883 & -1.994682 & -0.014977 \\ \mathrm{H} & 4.712369 & 0.126698 & 0.060155 \\ \mathrm{H} & 0.919041 & 1.935204 & 0.937271 \\ \mathrm{H} & 3.371402 & 2.037564 & 0.977165 \\ \mathrm{H} & 3.328004 & -1.263726 & -1.543471 \\ \mathrm{H} & -0.919052 & 1.935213 & -0.937258 \\ \mathrm{H} & -0.977536 & -1.949193 & 0.948005 \\ \mathrm{H} & -3.330897 & -1.994693 & 0.015019 \\ \mathrm{H} & -4.712370 & 0.126704 & -0.060095 \\ \mathrm{H} & -3.371415 & 2.037581 & -0.977104 \\ \mathrm{H} & -3.327968 & -1.263706 & 1.543499 \\ \mathrm{H} & & & \end{array}$

\begin{tabular}{lrrr} 
Fig3 : Bz22+-VI & & \\
24 E (B3LYP/CC-pVDZ) & \multicolumn{3}{c}{$-463.829581120 \mathrm{a} . \mathrm{u}}$. \\
& & & \\
$\mathrm{C}$ & 1.474321 & 1.149490 & 0.401111 \\
$\mathrm{C}$ & 0.727094 & -0.006396 & 0.000545 \\
$\mathrm{C}$ & 1.446100 & -1.122710 & -0.385918 \\
$\mathrm{C}$ & 2.914622 & -1.124698 & -0.399406 \\
$\mathrm{C}$ & 3.601513 & 0.096875 & 0.025840 \\
$\mathrm{C}$ & 2.877280 & 1.205749 & 0.418795 \\
$\mathrm{C}$ & -0.761758 & 0.030577 & -0.008017 \\
$\mathrm{C}$ & -1.540054 & -1.162784 & 0.423581 \\
$\mathrm{C}$ & -3.010915 & -1.078906 & 0.365011 \\
$\mathrm{C}$ & -3.651867 & 0.069181 & -0.039230 \\
$\mathrm{C}$ & -2.867084 & 1.177267 & -0.415471 \\
$\mathrm{C}$ & -1.448334 & 1.162751 & -0.396020 \\
$\mathrm{H}$ & 0.947993 & -2.035578 & -0.723220 \\
$\mathrm{H}$ & 3.298679 & -1.996853 & 0.185954 \\
$\mathrm{H}$ & 4.695953 & 0.108822 & 0.027405 \\
$\mathrm{H}$ & 0.927459 & 2.037090 & 0.733497 \\
$\mathrm{H}$ & 3.377564 & 2.119035 & 0.746261 \\
$\mathrm{H}$ & 3.283326 & -1.431069 & -1.409974 \\
$\mathrm{H}$ & -0.922409 & 2.058179 & -0.732835 \\
$\mathrm{H}$ & -1.214357 & -2.087902 & -0.098560 \\
$\mathrm{H}$ & -3.580140 & -1.962687 & 0.669035 \\
$\mathrm{H}$ & -4.741074 & 0.128712 & -0.074093 \\
$\mathrm{H}$ & -3.368457 & 2.092233 & -0.746834 \\
$\mathrm{H}$ & -1.270050 & -1.408355 & 1.478431 \\
& & & \\
& & &
\end{tabular}


Fig3 : $\mathrm{Bz}_{2}{ }^{2+}-\mathrm{VII}$

$24 \mathrm{E}(\mathrm{B} 3 \mathrm{LYP} / \mathrm{CC}-\mathrm{pVDZ})=-463.829395789 \mathrm{a} \cdot \mathrm{u}$.

$\begin{array}{lrrr}\mathrm{C} & 1.459450 & -1.160379 & -0.419698 \\ \mathrm{C} & 0.762145 & -0.036571 & -0.027134 \\ \mathrm{C} & 1.528912 & 1.162443 & 0.411601 \\ \mathrm{C} & 3.001151 & 1.079133 & 0.388133 \\ \mathrm{C} & 3.652831 & -0.064702 & -0.010496 \\ \mathrm{C} & 2.878298 & -1.171348 & -0.411773 \\ \mathrm{C} & -0.727331 & -0.019083 & -0.014916 \\ \mathrm{C} & -1.453703 & -1.126542 & 0.389305 \\ \mathrm{C} & -2.921154 & -1.119614 & 0.405322 \\ \mathrm{C} & -3.601559 & 0.103497 & -0.025855 \\ \mathrm{C} & -2.870382 & 1.205552 & -0.424237 \\ \mathrm{C} & -1.466906 & 1.140452 & -0.410477 \\ \mathrm{H} & -0.951973 & -2.035175 & 0.733649 \\ \mathrm{H} & -3.305868 & -1.991267 & -0.181583 \\ \mathrm{H} & -3.293935 & -1.427457 & 1.413540 \\ \mathrm{H} & -0.923154 & 2.029157 & -0.744381 \\ \mathrm{H} & -3.365273 & 2.121251 & -0.753005 \\ \mathrm{H} & -4.695839 & 0.122724 & -0.026615 \\ \mathrm{H} & 1.224602 & 1.441799 & 1.447371 \\ \mathrm{H} & 0.939601 & -2.051999 & -0.775174 \\ \mathrm{H} & 3.388477 & -2.081866 & -0.742014 \\ \mathrm{H} & 4.742678 & -0.121239 & -0.024375 \\ \mathrm{H} & 3.561808 & 1.963785 & 0.705299 \\ \mathrm{H} & 1.228372 & 2.073264 & -0.151376 \\ & & & \end{array}$

$24 \mathrm{E}(\mathrm{B} 3 \mathrm{LYP} / \mathrm{CC}-\mathrm{pVDZ})=-463.960313148 \mathrm{a} \cdot \mathrm{u}$.

$\begin{array}{lrrr}\mathrm{C} & 1.451909 & -1.150835 & -0.423971 \\ \mathrm{C} & 0.758893 & -0.037500 & -0.027030 \\ \mathrm{C} & 1.522760 & 1.154657 & 0.416973 \\ \mathrm{C} & 2.989150 & 1.070249 & 0.391641 \\ \mathrm{C} & 3.634197 & -0.064335 & -0.012067 \\ \mathrm{C} & 2.863080 & -1.162323 & -0.416996 \\ \mathrm{C} & -0.725177 & -0.019937 & -0.014760 \\ \mathrm{C} & -1.445945 & -1.117775 & 0.393523 \\ \mathrm{C} & -2.907492 & -1.111748 & 0.410900 \\ \mathrm{C} & -3.583636 & 0.104688 & -0.025599 \\ \mathrm{C} & -2.856432 & 1.196354 & -0.428786 \\ \mathrm{C} & -1.460908 & 1.131175 & -0.415138 \\ \mathrm{H} & -0.948973 & -2.015963 & 0.738085 \\ \mathrm{H} & -3.288707 & -1.978377 & -0.167304 \\ \mathrm{H} & -3.276129 & -1.409587 & 1.412891 \\ \mathrm{H} & -0.921600 & 2.008319 & -0.752216 \\ \mathrm{H} & -3.346350 & 2.101379 & -0.759920 \\ \mathrm{H} & -4.666838 & 0.123109 & -0.025298 \\ \mathrm{H} & 1.220548 & 1.426791 & 1.444762 \\ \mathrm{H} & 0.936027 & -2.031762 & -0.780490 \\ \mathrm{H} & 3.368005 & -2.062213 & -0.749085 \\ \mathrm{H} & 4.713457 & -0.121570 & -0.027224 \\ \mathrm{H} & 3.543984 & 1.944297 & 0.710813 \\ \mathrm{H} & 1.224192 & 2.059567 & -0.137161\end{array}$


Fig3 : $\mathrm{Bz}_{2}{ }^{2+}-$ VIII

$\mathrm{E}(\mathrm{B} 3 \mathrm{LYP} / \mathrm{cC}-\mathrm{pVDZ})=-463.830114767 \mathrm{a} \cdot \mathrm{u}$.

$\begin{array}{lrrr}\mathrm{C} & -1.488104 & 1.104712 & -0.503892 \\ \mathrm{C} & -0.750040 & -0.008701 & 0.001555 \\ \mathrm{C} & -1.467880 & -1.082432 & 0.488665 \\ \mathrm{C} & -2.936962 & -1.083701 & 0.493367 \\ \mathrm{C} & -3.620004 & 0.097648 & -0.039505 \\ \mathrm{C} & -2.893028 & 1.165464 & -0.526020 \\ \mathrm{C} & 0.750040 & 0.008628 & 0.001593 \\ \mathrm{C} & 1.467851 & 1.082402 & 0.488651 \\ \mathrm{C} & 2.936933 & 1.083775 & 0.493210 \\ \mathrm{C} & 3.620007 & -0.097617 & -0.039524 \\ \mathrm{C} & 2.893060 & -1.165473 & -0.525994 \\ \mathrm{C} & 1.488133 & -1.104808 & -0.503760 \\ \mathrm{H} & 0.963753 & 1.961096 & 0.902259 \\ \mathrm{H} & 3.316849 & 2.000394 & -0.022433 \\ \mathrm{H} & 3.313399 & 1.304647 & 1.522343 \\ \mathrm{H} & 0.935644 & -1.958200 & -0.910373 \\ \mathrm{H} & 3.390136 & -2.048177 & -0.932326 \\ \mathrm{H} & 4.714209 & -0.111451 & -0.045193 \\ \mathrm{H} & -0.963805 & -1.961113 & 0.902328 \\ \mathrm{H} & -0.935589 & 1.958163 & -0.910348 \\ \mathrm{H} & -3.390082 & 2.048198 & -0.932314 \\ \mathrm{H} & -4.714206 & 0.111530 & -0.045136 \\ \mathrm{H} & -3.313178 & -1.303933 & 1.522757 \\ \mathrm{H} & -3.317158 & -2.000535 & -0.021629\end{array}$

$\mathrm{E}(\mathrm{B} 3 \mathrm{LYP} / \mathrm{cC}-\mathrm{pVTZ})=-463.960852986 \mathrm{a} \cdot \mathrm{u} \cdot$

$\begin{array}{lrrr}\mathrm{C} & 1.482614 & -1.100927 & -0.494046 \\ \mathrm{C} & 0.747700 & 0.009460 & 0.001398 \\ \mathrm{C} & 1.459858 & 1.079286 & 0.478551 \\ \mathrm{C} & 2.923111 & 1.081930 & 0.484546 \\ \mathrm{C} & 3.602358 & -0.098397 & -0.038740 \\ \mathrm{C} & 2.879489 & -1.161684 & -0.515707 \\ \mathrm{C} & -0.747700 & -0.009456 & 0.001374 \\ \mathrm{C} & -1.459874 & -1.079317 & 0.478425 \\ \mathrm{C} & -2.923127 & -1.081981 & 0.484332 \\ \mathrm{C} & -3.602357 & 0.098416 & -0.038820 \\ \mathrm{C} & -2.879473 & 1.161735 & -0.515693 \\ \mathrm{C} & -1.482599 & 1.100972 & -0.494002 \\ \mathrm{H} & -0.961304 & -1.952425 & 0.882036 \\ \mathrm{H} & -3.299448 & -1.987042 & -0.033329 \\ \mathrm{H} & -3.295275 & -1.305664 & 1.503878 \\ \mathrm{H} & -0.935248 & 1.948215 & -0.891878 \\ \mathrm{H} & -3.371569 & 2.038544 & -0.912635 \\ \mathrm{H} & -4.685483 & 0.111191 & -0.043452 \\ \mathrm{H} & 0.961275 & 1.952362 & 0.882214 \\ \mathrm{H} & 0.935276 & -1.948153 & -0.891976 \\ \mathrm{H} & 3.371598 & -2.038459 & -0.912709 \\ \mathrm{H} & 4.685485 & -0.111164 & -0.043357 \\ \mathrm{H} & 3.295116 & 1.305247 & 1.504240 \\ \mathrm{H} & 3.299559 & 1.987137 & -0.032737 \\ & & & \end{array}$


Fig3 : $\mathrm{Bz}_{2}{ }^{2+}-\mathrm{IX}$

$24 \mathrm{E}(\mathrm{B} 3 \mathrm{LYP} / \mathrm{CC}-\mathrm{PVDZ})=-463.831450848 \mathrm{a} \cdot \mathrm{u}$.

$\begin{array}{lrrr}\mathrm{C} & -1.440196 & -1.128316 & 0.515074 \\ \mathrm{C} & -0.747117 & -0.000037 & -0.000031 \\ \mathrm{C} & -1.440152 & 1.128285 & -0.515099 \\ \mathrm{C} & -2.818208 & 1.134796 & -0.524965 \\ \mathrm{C} & -3.581976 & 0.000026 & -0.000016 \\ \mathrm{C} & -2.818253 & -1.134773 & 0.524938 \\ \mathrm{C} & 0.747117 & -0.000035 & 0.000024 \\ \mathrm{C} & 1.440150 & 1.128286 & 0.515097 \\ \mathrm{C} & 2.818206 & 1.134796 & 0.524969 \\ \mathrm{C} & 3.581976 & 0.000027 & 0.000020 \\ \mathrm{C} & 2.818255 & -1.134773 & -0.524934 \\ \mathrm{C} & 1.440198 & -1.128316 & -0.515076 \\ \mathrm{H} & -0.885403 & 1.975612 & -0.921718 \\ \mathrm{H} & -3.364894 & 1.993838 & -0.925193 \\ \mathrm{H} & -4.315905 & -0.356029 & -0.766067 \\ \mathrm{H} & -0.885483 & -1.975648 & 0.921729 \\ \mathrm{H} & -3.364974 & -1.993788 & 0.925176 \\ \mathrm{H} & -4.315876 & 0.356116 & 0.766048 \\ \mathrm{H} & 0.885486 & -1.975649 & -0.921731 \\ \mathrm{H} & 0.885399 & 1.975611 & 0.921717 \\ \mathrm{H} & 3.364891 & 1.993837 & 0.925201 \\ \mathrm{H} & 4.315863 & 0.356123 & -0.766055 \\ \mathrm{H} & 3.364977 & -1.993790 & -0.925168 \\ \mathrm{H} & 4.315918 & -0.356021 & 0.766060\end{array}$

24 E (B3LYP/CC-PVTZ)

$=-463.962480562 \mathrm{a} \cdot \mathrm{u}$.

$\begin{array}{lrrr}\mathrm{C} & -1.433788 & -1.121802 & 0.512959 \\ \mathrm{C} & -0.744438 & -0.000102 & -0.000083 \\ \mathrm{C} & -1.433681 & 1.121717 & -0.513001 \\ \mathrm{C} & -2.802710 & 1.129232 & -0.523360 \\ \mathrm{C} & -3.563997 & 0.000069 & 0.000000 \\ \mathrm{C} & -2.802817 & -1.129171 & 0.523352 \\ \mathrm{C} & 0.744438 & -0.000094 & 0.000045 \\ \mathrm{C} & 1.433671 & 1.121720 & 0.512988 \\ \mathrm{C} & 2.802700 & 1.129235 & 0.523373 \\ \mathrm{C} & 3.563997 & 0.000073 & 0.000025 \\ \mathrm{C} & 2.802827 & -1.129168 & -0.523338 \\ \mathrm{C} & 1.433797 & -1.121799 & -0.512972 \\ \mathrm{H} & -0.882902 & 1.960405 & -0.914853 \\ \mathrm{H} & -3.344781 & 1.979073 & -0.919367 \\ \mathrm{H} & -4.290701 & -0.353699 & -0.759881 \\ \mathrm{H} & -0.883093 & -1.960503 & 0.914897 \\ \mathrm{H} & -3.344970 & -1.978939 & 0.919403 \\ \mathrm{H} & -4.290621 & 0.353923 & 0.759919 \\ \mathrm{H} & 0.883110 & -1.960505 & -0.914910 \\ \mathrm{H} & 0.882885 & 1.960403 & 0.914841 \\ \mathrm{H} & 3.344765 & 1.979073 & 0.919397 \\ \mathrm{H} & 4.290616 & 0.353935 & -0.759896 \\ \mathrm{H} & 3.344986 & -1.978940 & -0.919373 \\ \mathrm{H} & 4.290705 & -0.353686 & 0.759904\end{array}$


Fig5 : Biphenyl in neutral form

$22 \mathrm{E}(\mathrm{B} 3 \mathrm{LYP} / \mathrm{cC}-\mathrm{pVDZ})=-463.333573855 \mathrm{a} \cdot \mathrm{u}$.

$\begin{array}{lrrr}\mathrm{C} & 0.000000 & 0.000000 & 0.743676 \\ \mathrm{C} & 0.000000 & 0.000000 & -0.743676 \\ \mathrm{C} & -0.402516 & 1.137604 & 1.468246 \\ \mathrm{C} & -0.402516 & -1.137604 & -1.468246 \\ \mathrm{C} & 0.402516 & -1.137604 & 1.468246 \\ \mathrm{C} & 0.402516 & 1.137604 & -1.468246 \\ \mathrm{C} & -0.402781 & 1.137837 & 2.864532 \\ \mathrm{C} & 0.402781 & -1.137837 & 2.864532 \\ \mathrm{C} & -0.402781 & -1.137837 & -2.864532 \\ \mathrm{C} & 0.402781 & 1.137837 & -2.864532 \\ \mathrm{C} & 0.000000 & 0.000000 & 3.569926 \\ \mathrm{C} & 0.000000 & 0.000000 & -3.569926 \\ \mathrm{H} & -0.744110 & 2.024111 & 0.929549 \\ \mathrm{H} & 0.744110 & -2.024111 & 0.929549 \\ \mathrm{H} & -0.744110 & -2.024111 & -0.929549 \\ \mathrm{H} & 0.744110 & 2.024111 & -0.929549 \\ \mathrm{H} & -0.727765 & 2.030448 & 3.404403 \\ \mathrm{H} & -0.727765 & -2.030448 & -3.404403 \\ \mathrm{H} & 0.727765 & 2.030448 & -3.404403 \\ \mathrm{H} & 0.727765 & -2.030448 & 3.404403 \\ \mathrm{H} & 0.000000 & 0.000000 & 4.662218 \\ \mathrm{H} & 0.000000 & 0.000000 & -4.662218\end{array}$

E (B3LYP/CC-pVTZ)

$$
=
$$$$
-463.466425267 \text { a.u. }
$$

$$
\begin{aligned}
& 0.000000 \\
& 0.000000
\end{aligned}
$$

0.000000

0.000000

$-0.402031$

$-0.402031$

0.402031

0.402031

$-0.402658$

0.402658

$-0.402658$

0.402658

0.000000

0.000000

$-0.740777$

0.740777

$-0.740777$

0.740777

$-0.726205$

$-0.726205$

0.726205

0.726205

0.000000

0.000000
0.741126

$-0.741126$

1. 461742

$-1.461742$

$-1.461742$

2.850157

2.850157

$-2.850157$

$-2.850157$

3. 551397

$-3.551397$

0.928419

0.928419

$-0.928419$

$-0.928419$

3.385216

$-3.385216$

$-3.385216$

3. 385216

4.633086

$-4.633086$
1. 461742 
Fig6 : $\left[\mathrm{C}_{6} \mathrm{~F}_{6}\right]_{2}{ }^{2+}-\mathrm{I}$

$24 \mathrm{E}\left(\mathrm{B} 3 \mathrm{LYP} / 6-31+\mathrm{G}^{*}\right)=-1654.46791765 \mathrm{a} \cdot \mathrm{u}$.

\begin{tabular}{|c|c|c|c|}
\hline $\mathrm{C}$ & -1.216001 & 1.052732 & 0.766085 \\
\hline $\mathrm{C}$ & -0.613918 & -0.332646 & 0.548671 \\
\hline $\mathrm{C}$ & -1.672648 & -1.357360 & 0.123944 \\
\hline $\mathrm{C}$ & -2.951134 & -1.012161 & -0.207634 \\
\hline $\mathrm{C}$ & -3.360812 & 0.348076 & -0.053253 \\
\hline $\mathrm{C}$ & -2.493547 & 1.381881 & 0.440128 \\
\hline $\mathrm{C}$ & 0.613896 & -0.332625 & -0.548401 \\
\hline $\mathrm{C}$ & 1.672629 & -1.357364 & -0.123849 \\
\hline $\mathrm{C}$ & 2.951211 & -1.012131 & 0.207342 \\
\hline $\mathrm{C}$ & 3.360917 & 0.348098 & 0.052781 \\
\hline $\mathrm{C}$ & 2.493596 & 1.381968 & -0.440210 \\
\hline $\mathrm{C}$ & 1.215874 & 1.052914 & -0.765598 \\
\hline $\mathrm{F}$ & 2.986788 & 2.581750 & -0.616246 \\
\hline $\mathrm{F}$ & 4.570570 & 0.656379 & 0.330785 \\
\hline $\mathrm{F}$ & 3.837714 & -1.894846 & 0.593029 \\
\hline $\mathrm{F}$ & 1.290806 & -2.595956 & -0.083761 \\
\hline $\mathrm{F}$ & 0.070202 & -0.721611 & -1.748229 \\
\hline $\mathrm{F}$ & 0.404551 & 1.920734 & -1.310929 \\
\hline $\mathrm{F}$ & -0.070477 & -0.721862 & 1.748617 \\
\hline $\mathrm{F}$ & -1.290799 & -2.595953 & 0.08402 \\
\hline $\mathrm{F}$ & -3.837469 & -1.894943 & -0.59352 \\
\hline F & -4.570340 & 0.656466 & -0.33161 \\
\hline $\mathrm{F}$ & -2.986727 & 2.581695 & 0.61607 \\
\hline $\mathrm{F}$ & -0.404861 & 1.920563 & 1.31176 \\
\hline
\end{tabular}

Fig6 : $\left[\mathrm{C}_{6} \mathrm{~F}_{6}\right]_{2}{ }^{2+}-\mathrm{II}$

$24 \mathrm{E}\left(\mathrm{B} 3 \mathrm{LYP} / 6-31+\mathrm{G}^{*}\right)=-1654.46486428 \mathrm{a} \cdot \mathrm{u}$.

$\begin{array}{lrrr}C & 2.996995 & 0.365677 & 0.426804 \\ C & 2.971652 & -0.863644 & -0.292059 \\ C & 1.865862 & -1.279894 & -1.116735 \\ C & 0.787234 & -0.461080 & -1.220581 \\ C & 0.663294 & 0.859545 & -0.477569 \\ C & 1.921573 & 1.208607 & 0.325629 \\ C & -0.663318 & 0.859658 & 0.477487 \\ C & -1.921599 & 1.208627 & -0.325749 \\ C & -2.996941 & 0.365597 & -0.426965 \\ C & -2.971571 & -0.863654 & 0.292020 \\ C & -1.865874 & -1.279701 & 1.116922 \\ C & -0.787285 & -0.460837 & 1.220729 \\ \text { F } & 0.244087 & -0.766543 & 1.973401 \\ \text { F } & -0.504543 & 1.859122 & 1.399116 \\ \text { F } & -1.959184 & -2.421608 & 1.751238 \\ \text { F } & -3.992589 & -1.633272 & 0.228088 \\ \text { F } & -4.075526 & 0.686386 & -1.094832 \\ \text { F } & -1.951973 & 2.389974 & -0.847168 \\ \text { F } & 3.992726 & -1.633188 & -0.228130 \\ \text { F } & 4.075593 & 0.686593 & 1.094587 \\ \text { F } & 1.951846 & 2.389940 & 0.847091 \\ \text { F } & 0.504602 & 1.858865 & -1.399372 \\ \text { F } & -0.244186 & -0.766951 & -1.973125 \\ \text { F } & 1.959131 & -2.421916 & -1.750850\end{array}$




\begin{tabular}{|c|c|c|c|}
\hline 24 & $\mathrm{E}(\mathrm{B} 3 \mathrm{LYP} / 6-31+\mathrm{G} * *)$ & -1654.45 & 3533 a.u. \\
\hline $\mathrm{C}$ & 2.640773 & 0.054455 & 0.327507 \\
\hline C & 2.329515 & -1.153721 & -0.312728 \\
\hline$C$ & 1.352768 & -1.213405 & -1.392583 \\
\hline C & 0.729511 & -0.058392 & -1.850902 \\
\hline C & 1.030897 & 1.153951 & -1.210835 \\
\hline $\mathrm{C}$ & 2.001433 & 1.208895 & -0.120516 \\
\hline $\mathrm{C}$ & -1.025837 & 1.146673 & 1.219604 \\
\hline $\mathrm{C}$ & -1.994728 & 1.213343 & 0.128223 \\
\hline $\mathrm{C}$ & -2.638968 & 0.064886 & -0.328518 \\
\hline $\mathrm{C}$ & -2.334395 & -1.148856 & 0.303929 \\
\hline $\mathrm{C}$ & -1.359399 & -1.220132 & 1.384589 \\
\hline $\mathrm{C}$ & -0.731293 & -0.071412 & 1.851628 \\
\hline $\mathrm{F}$ & 0.145958 & -0.118251 & 2.832609 \\
\hline F & -0.529342 & 2.266434 & 1.657661 \\
\hline F & -1.127537 & -2.384263 & 1.914745 \\
\hline F & -2.893966 & -2.251658 & -0.072859 \\
\hline F & -3.507025 & 0.113927 & -1.308485 \\
\hline F & -2.225784 & 2.377359 & -0.396067 \\
\hline F & 2.884535 & -2.261314 & 0.056542 \\
\hline F & 3.510113 & 0.092451 & 1.306774 \\
\hline F & 2.238159 & 2.368371 & 0.411397 \\
\hline F & 0.539179 & 2.278904 & -1.640661 \\
\hline $\mathrm{F}$ & -0.149437 & -0.093150 & -2.830890 \\
\hline$F$ & 1.114965 & -2.372999 & -1.930364 \\
\hline
\end{tabular}

\begin{tabular}{lrrr} 
Fig7 : $\mathrm{Bz}_{3}{ }^{2+}-\mathrm{I}$ & \\
36 E (B3LYP/6-31+G**) & \multicolumn{3}{c}{$-696.071939476 \mathrm{a} . \mathrm{u}}$. \\
& & & \\
$\mathrm{C}$ & -3.121912 & 0.845447 & 0.662229 \\
$\mathrm{C}$ & -2.788201 & -0.582876 & 0.494487 \\
$\mathrm{C}$ & -3.574627 & -1.302782 & -0.528800 \\
$\mathrm{C}$ & -4.589565 & -0.693046 & -1.224038 \\
$\mathrm{C}$ & -4.869826 & 0.670764 & -0.979915 \\
$\mathrm{C}$ & -4.144432 & 1.436087 & -0.038409 \\
$\mathrm{C}$ & -1.221780 & -0.925303 & 0.473672 \\
$\mathrm{C}$ & -0.549635 & -0.496941 & 1.752030 \\
$\mathrm{C}$ & 0.542049 & 0.276988 & 1.794928 \\
$\mathrm{C}$ & 1.214141 & 0.843940 & 0.571700 \\
$\mathrm{C}$ & 0.547524 & 0.407986 & -0.708902 \\
$\mathrm{C}$ & -0.542891 & -0.366590 & -0.751884 \\
$\mathrm{H}$ & -0.966609 & -0.632787 & -1.719010 \\
$\mathrm{H}$ & 0.981261 & 0.765521 & -1.641550 \\
$\mathrm{H}$ & -1.204518 & -2.025307 & 0.424537 \\
$\mathrm{H}$ & -0.977543 & -0.862480 & 2.684766 \\
$\mathrm{H}$ & 0.971006 & 0.535701 & 2.762237 \\
$\mathrm{H}$ & 1.184743 & 1.942895 & 0.636783 \\
$\mathrm{H}$ & -3.124148 & -1.052451 & 1.450945 \\
$\mathrm{H}$ & -3.354286 & -2.356170 & -0.691251 \\
$\mathrm{H}$ & -5.177119 & -1.243247 & -1.952689 \\
$\mathrm{H}$ & -5.674963 & 1.147961 & -1.535174 \\
$\mathrm{H}$ & -4.397768 & 2.480473 & 0.115976 \\
& & &
\end{tabular}




\begin{tabular}{|c|c|c|c|}
\hline $\mathrm{H}$ & -2.549142 & 1.417542 & 1.388290 \\
\hline C & 2.785897 & 0.518350 & 0.566423 \\
\hline $\mathrm{C}$ & 3.569084 & 1.367803 & -0.354887 \\
\hline $\mathrm{C}$ & 4.586397 & 0.857676 & -1.122990 \\
\hline $\mathrm{C}$ & 4.874801 & -0.524589 & -1.054555 \\
\hline $\mathrm{C}$ & 4.155213 & -1.407732 & -0.217459 \\
\hline $\mathrm{C}$ & 3.130197 & -0.917056 & 0.553107 \\
\hline $\mathrm{H}$ & 3.342114 & 2.431881 & -0.382532 \\
\hline $\mathrm{H}$ & 5.169753 & 1.499442 & -1.776215 \\
\hline $\mathrm{H}$ & 5.681226 & -0.922813 & -1.667276 \\
\hline $\mathrm{H}$ & 4.415041 & -2.461694 & -0.197024 \\
\hline $\mathrm{H}$ & 2.561950 & -1.580589 & 1.200844 \\
\hline $\mathrm{H}$ & 3.104392 & 0.867367 & 1.577917 \\
\hline 36 & E (B3LYP/cC-pVDZ) & -696.09199 & 94160 \\
\hline C & 0.253302 & 0.017801 & -1.397793 \\
\hline $\mathrm{C}$ & 1.196943 & -0.756474 & -0.514925 \\
\hline $\mathrm{C}$ & 0.818105 & -0.680276 & 0.943433 \\
\hline C & -0.253353 & -0.017835 & 1.397891 \\
\hline C & -1.196984 & 0.756452 & 0.515026 \\
\hline C & -0.818154 & 0.680244 & -0.943337 \\
\hline C & 2.712219 & -0.319751 & -0.744939 \\
\hline C & 3.025486 & 1.094616 & -0.466134 \\
\hline C & 4.218159 & 1.470753 & 0.103980 \\
\hline $\mathrm{C}$ & 5.147894 & 0.475423 & 0.483787 \\
\hline C & 4.902336 & -0.903151 & 0.289444 \\
\hline C & 3.717869 & -1.298455 & -0.285033 \\
\hline $\mathrm{H}$ & 2.289336 & 1.847506 & -0.760961 \\
\hline $\mathrm{H}$ & 4.451323 & 2.523101 & 0.275012 \\
\hline $\mathrm{H}$ & 2.825508 & -0.382368 & -1.862821 \\
\hline $\mathrm{H}$ & 3.515693 & -2.360188 & -0.456352 \\
\hline $\mathrm{H}$ & 5.650017 & -1.637015 & 0.595230 \\
\hline $\mathrm{H}$ & 6.091615 & 0.783168 & 0.944753 \\
\hline $\mathrm{H}$ & 1.181009 & -1.815842 & -0.834876 \\
\hline $\mathrm{H}$ & 0.456252 & 0.008028 & -2.473690 \\
\hline $\mathrm{H}$ & -1.462287 & 1.204311 & -1.656432 \\
\hline $\mathrm{H}$ & -1.181046 & 1.815821 & 0.834983 \\
\hline $\mathrm{H}$ & -0.456307 & -0.008084 & 2.473788 \\
\hline $\mathrm{H}$ & 1.462225 & -1.204362 & 1.656525 \\
\hline $\mathrm{C}$ & -2.712264 & 0.319741 & 0.745043 \\
\hline C & -3.717892 & 1.298456 & 0.285125 \\
\hline C & -4.902248 & 0.903182 & -0.289597 \\
\hline C & -5.147752 & -0.475376 & -0.484100 \\
\hline C & -4.218104 & -1.470724 & -0.104136 \\
\hline $\mathrm{C}$ & -3.025551 & -1.094621 & 0.466249 \\
\hline $\mathrm{H}$ & -3.515825 & 2.360177 & 0.456654 \\
\hline $\mathrm{H}$ & -5.649893 & 1.637069 & -0.595414 \\
\hline $\mathrm{H}$ & -6.091394 & -0.783101 & -0.945244 \\
\hline $\mathrm{H}$ & -4.451247 & -2.523068 & -0.275216 \\
\hline $\mathrm{H}$ & -2.289495 & -1.847544 & 0.761232 \\
\hline $\mathrm{H}$ & -2.825541 & 0.382355 & 1.862927 \\
\hline
\end{tabular}

Fig7 : $\mathrm{Bz}_{3}{ }^{2+-} \mathrm{II}$

$36 \mathrm{E}\left(\mathrm{B} 3 \mathrm{LYP} / 6-31+\mathrm{G}^{* *}\right)=-696.081137511 \mathrm{a} \cdot \mathrm{u}$.

$\begin{array}{lrrr}\text { C } & 3.611605 & -1.251930 & 0.349688 \\ \text { C } & 2.892514 & 0.000716 & 0.692422\end{array}$




\begin{tabular}{lrrr} 
C & 3.611281 & 1.252741 & 0.346719 \\
$\mathrm{C}$ & 4.884371 & 1.239967 & -0.166879 \\
$\mathrm{C}$ & 5.513033 & -0.000259 & -0.423254 \\
$\mathrm{C}$ & 4.884690 & -1.240036 & -0.163942 \\
$\mathrm{C}$ & 1.387052 & 0.000139 & 0.367294 \\
$\mathrm{C}$ & 0.469509 & 0.001562 & 1.341476 \\
$\mathrm{C}$ & -1.017422 & 0.001259 & 1.100886 \\
$\mathrm{C}$ & -1.386931 & -0.000275 & -0.366973 \\
$\mathrm{C}$ & -0.469385 & -0.001731 & -1.341156 \\
$\mathrm{C}$ & 1.017548 & -0.001927 & -1.100578 \\
$\mathrm{H}$ & 1.460074 & 0.869391 & -1.610019 \\
$\mathrm{H}$ & -0.773215 & -0.002836 & -2.387068 \\
$\mathrm{H}$ & 1.459442 & -0.875093 & -1.607404 \\
$\mathrm{H}$ & 0.773330 & 0.003049 & 2.387389 \\
$\mathrm{H}$ & -1.459590 & -0.870496 & 1.609903 \\
$\mathrm{H}$ & -1.459670 & 0.873977 & 1.608140 \\
$\mathrm{H}$ & 2.946083 & 0.002058 & 1.810339 \\
$\mathrm{H}$ & 3.108239 & 2.193359 & 0.559942 \\
$\mathrm{H}$ & 5.410238 & 2.165461 & -0.381181 \\
$\mathrm{H}$ & 6.521454 & -0.000617 & -0.832612 \\
$\mathrm{H}$ & 5.410808 & -2.165901 & -0.376018 \\
$\mathrm{H}$ & 3.108827 & -2.192172 & 0.565182 \\
$\mathrm{C}$ & -2.892366 & -0.000309 & -0.692061 \\
$\mathrm{C}$ & -3.611326 & 1.252184 & -0.348515 \\
$\mathrm{C}$ & -4.884654 & 1.240092 & 0.164513 \\
$\mathrm{C}$ & -5.513327 & 0.000213 & 0.422532 \\
$\mathrm{C}$ & -4.884748 & -1.239902 & 0.165451 \\
$\mathrm{C}$ & -3.611413 & -1.252476 & -0.347564 \\
$\mathrm{H}$ & -3.108285 & 2.192511 & -0.563022 \\
$\mathrm{H}$ & -5.410703 & 2.165874 & 0.377119 \\
$\mathrm{H}$ & -6.521947 & 0.000412 & 0.831397 \\
$\mathrm{H}$ & -5.410842 & -2.165487 & 0.378799 \\
$\mathrm{H}$ & -3.108414 & -2.193014 & -0.561240 \\
$\mathrm{H}$ & -2.946005 & -0.000656 & -1.810002 \\
\hline & & &
\end{tabular}

Fig8 : $\left[\mathrm{C}_{6} \mathrm{~F}_{6}\right]_{3}{ }^{2+}-\mathrm{I}$

$36 \mathrm{E}\left(\mathrm{B} 3 \mathrm{LYP} / 6-31+\mathrm{G}^{* *}\right)=-2482.14451865 \mathrm{a} \cdot \mathrm{u}$.

$\begin{array}{lrrr}\text { C } & 0.024452 & 0.179730 & -0.021998 \\ \text { C } & 0.047132 & 0.061814 & 1.499503 \\ \text { C } & 1.466415 & 0.010364 & 2.042179 \\ \text { C } & 2.563642 & -0.182590 & 1.261181 \\ \text { C } & 2.391591 & -0.208410 & -0.156993 \\ \text { C } & 1.132732 & -0.024252 & -0.794597 \\ \text { C } & -0.844939 & -1.251465 & 1.955177 \\ \text { C } & -0.286609 & -1.973374 & 3.149564 \\ \text { C } & -0.935950 & -2.148714 & 4.303279 \\ \text { C } & -2.353596 & -1.720921 & 4.560357 \\ \text { C } & -2.912978 & -0.989980 & 3.369997 \\ \text { C } & -2.266671 & -0.827543 & 2.206458 \\ \text { C } & -3.358000 & -2.969842 & 4.960175 \\ \text { C } & -2.592450 & -4.188964 & 5.449946 \\ \text { C } & -2.616126 & -4.624121 & 6.738811 \\ \text { C } & -3.506082 & -3.984024 & 7.655170 \\ \text { C } & -4.382421 & -2.926183 & 7.283429 \\ \text { C } & -4.375137 & -2.486010 & 5.990015 \\ \text { F } & -1.895157 & -4.808084 & 4.536007 \\ \text { F } & -4.014658 & -3.344840 & 3.814742\end{array}$




$\begin{array}{lrrr}F & -5.234747 & -1.599108 & 5.595206 \\ F & -5.225678 & -2.460875 & 8.178015 \\ F & -3.545772 & -4.416078 & 8.866156 \\ F & -1.908871 & -5.650570 & 7.156827 \\ F & -2.348781 & -0.899490 & 5.682041 \\ F & -0.338748 & -2.740010 & 5.337849 \\ F & 0.947403 & -2.449173 & 2.983832 \\ F & -0.776414 & -2.117874 & 0.869993 \\ F & -2.872848 & -0.226965 & 1.194252 \\ F & -4.143330 & -0.522036 & 3.511363 \\ F & -0.550367 & 1.168118 & 2.050080 \\ F & 1.570550 & 0.168348 & 3.333988 \\ F & 3.779687 & -0.273859 & 1.752524 \\ F & 3.435021 & -0.363730 & -0.893074 \\ F & 1.090202 & 0.021520 & -2.107622 \\ F & -1.116343 & 0.455755 & -0.573095\end{array}$

Fig8 : $\left[\mathrm{C}_{6} \mathrm{~F}_{6}\right]_{3}{ }^{2+}-\mathrm{II}$

$36 \mathrm{E}\left(\mathrm{B} 3 \mathrm{LYP} / 6-31+\mathrm{G}^{* *}\right)=-2482.13946073 \mathrm{a} \cdot \mathrm{u}$.

$\begin{array}{lrrr}C & -0.473406 & -0.003338 & 0.028138 \\ C & -0.236962 & 0.008746 & 1.511581 \\ C & 1.235868 & 0.164718 & 1.821627 \\ C & 2.171185 & 0.328332 & 0.879213 \\ C & 1.934643 & 0.316646 & -0.604251 \\ C & 0.461931 & 0.160026 & -0.914299 \\ C & -0.891266 & -1.292490 & 2.225017 \\ C & -0.429304 & -2.560652 & 1.528300 \\ C & -1.278181 & -3.430190 & 0.919226 \\ C & -2.689423 & -3.189660 & 1.003717 \\ C & -3.255749 & -2.084619 & 1.691056 \\ C & -2.416013 & -1.190862 & 2.298996 \\ F & -4.563388 & -1.985337 & 1.769963 \\ F & -3.478758 & -4.041469 & 0.451087 \\ F & -0.864842 & -4.508506 & 0.291105 \\ F & 0.864196 & -2.761722 & 1.531278 \\ F & -0.450050 & -1.328955 & 3.524188 \\ F & -2.890725 & -0.234324 & 3.029205 \\ F & -0.888323 & 1.094849 & 2.074225 \\ F & 1.555941 & 0.250509 & 3.100820 \\ F & 3.448934 & 0.536284 & 1.227134 \\ F & 2.586516 & -0.768882 & -1.167279 \\ F & 0.141990 & 0.073907 & -2.193502 \\ F & -1.751122 & -0.211554 & -0.319911 \\ C & 2.588063 & 1.618656 & -1.317267 \\ C & 4.112797 & 1.517827 & -1.391583 \\ C & 4.952256 & 2.411632 & -0.783273 \\ C & 4.385581 & 3.516104 & -0.095342 \\ C & 2.974211 & 3.755755 & -0.010308 \\ C & 2.125630 & 2.886182 & -0.619793 \\ F & 4.587943 & 0.561920 & -2.122330 \\ F & 6.259906 & 2.312932 & -0.862504 \\ F & 5.174616 & 4.368141 & 0.457381 \\ F & 2.560342 & 4.833328 & 0.618686 \\ F & 0.832022 & 3.086776 & -0.622165 \\ F & 2.146569 & 1.655278 & -2.616383\end{array}$




\begin{tabular}{lrrr} 
Fig $9: \mathrm{NaPh}_{2}{ }^{2+}$ & & \\
36 E (B3LYP/6-31+G**) & \multicolumn{3}{c}{$-771.139392506 \mathrm{a} . \mathrm{u}}$. \\
& & & \\
$\mathrm{C}$ & 1.103795 & -1.252284 & -1.083277 \\
$\mathrm{C}$ & 1.484551 & -0.062963 & -0.477028 \\
$\mathrm{C}$ & 2.751295 & -0.022509 & 0.201047 \\
$\mathrm{C}$ & 3.541471 & -1.203203 & 0.322647 \\
$\mathrm{C}$ & 3.123116 & -2.383761 & -0.269053 \\
$\mathrm{C}$ & 1.920400 & -2.396388 & -0.992280 \\
$\mathrm{C}$ & 0.644497 & 1.184426 & -0.550637 \\
$\mathrm{C}$ & 1.411993 & 2.440096 & -0.292757 \\
$\mathrm{C}$ & 2.643530 & 2.447170 & 0.294306 \\
$\mathrm{C}$ & 3.281515 & 1.215361 & 0.600084 \\
$\mathrm{C}$ & -0.644898 & 1.184658 & 0.551228 \\
$\mathrm{C}$ & -1.412462 & 2.440211 & 0.292970 \\
$\mathrm{C}$ & -1.104433 & -1.251736 & 1.084761 \\
$\mathrm{C}$ & -1.484871 & -0.062751 & 0.477719 \\
$\mathrm{C}$ & -1.920592 & -2.396110 & 0.993073 \\
$\mathrm{H}$ & 4.248911 & 1.235987 & 1.099204 \\
$\mathrm{H}$ & 4.492794 & -1.152894 & 0.845909 \\
$\mathrm{H}$ & 3.152592 & 3.381880 & 0.508983 \\
$\mathrm{H}$ & 3.731167 & -3.280439 & -0.202587 \\
$\mathrm{H}$ & 0.947910 & 3.385848 & -0.557133 \\
$\mathrm{H}$ & 1.613230 & -3.305621 & -1.502326 \\
$\mathrm{H}$ & 0.149827 & 1.245776 & -1.527186 \\
$\mathrm{H}$ & 0.187135 & -1.306165 & -1.662988 \\
$\mathrm{H}$ & -0.150270 & 1.246235 & 1.527761 \\
$\mathrm{H}$ & -0.948688 & 3.386059 & 0.557551 \\
$\mathrm{H}$ & -0.188440 & -1.305071 & 1.665552 \\
$\mathrm{H}$ & -1.613818 & -3.305113 & 1.503768 \\
$\mathrm{C}$ & -2.643748 & 2.446991 & -0.294621 \\
$\mathrm{C}$ & -2.750849 & -0.022744 & -0.201822 \\
$\mathrm{C}$ & -3.122446 & -2.383967 & 0.268510 \\
$\mathrm{C}$ & -3.281177 & 1.215035 & -0.601028 \\
$\mathrm{C}$ & -3.540632 & -1.203679 & -0.323801 \\
$\mathrm{H}$ & -3.152969 & 3.381596 & -0.509414 \\
$\mathrm{H}$ & -4.248302 & 1.235533 & -1.100679 \\
$\mathrm{H}$ & -4.491365 & -1.153810 & -0.848166 \\
$\mathrm{H}$ & -3.730037 & -3.280910 & 0.201499
\end{tabular}

$\begin{array}{lrrr}\text { Fig10 : } \mathrm{Naph}_{3}{ }^{2+}-\mathrm{I} \\ \text { 54 E (RHF/6-31G) }=-1149.02731993 & \mathrm{a} . \mathrm{u} . \\ \text { C } & 4.716779 & -0.250171 & -1.335785 \\ \text { C } & 4.103785 & -0.066510 & -0.105611 \\ \text { C } & 4.263731 & -1.068400 & 0.884023 \\ \text { C } & 5.006062 & -2.239300 & 0.601637 \\ \text { C } & 5.590840 & -2.406161 & -0.626864 \\ \text { C } & 5.449709 & -1.401699 & -1.590563 \\ \text { C } & 3.727665 & -0.859641 & 2.166209 \\ \text { C } & 3.044000 & 0.313380 & 2.533917 \\ \text { C } & 2.859272 & 1.287679 & 1.609255 \\ \text { C } & 3.323947 & 1.183581 & 0.205262 \\ \text { C } & 2.092928 & 1.465152 & -0.800588 \\ \text { C } & 0.887935 & 0.566178 & -0.528321 \\ \text { C } & -0.298957 & 1.149798 & 0.131156\end{array}$




\begin{tabular}{|c|c|c|c|}
\hline $\mathrm{C}$ & -0.363599 & 2.474870 & 0.331820 \\
\hline $\mathrm{C}$ & 0.661596 & 3.389684 & -0.167652 \\
\hline $\mathrm{C}$ & 1.775113 & 2.936470 & -0.732674 \\
\hline $\mathrm{C}$ & 0.907519 & -0.736631 & -0.840876 \\
\hline $\mathrm{C}$ & -0.158138 & -1.651306 & -0.433658 \\
\hline $\mathrm{C}$ & -1.232157 & -1.222285 & 0.217588 \\
\hline $\mathrm{C}$ & -1.478037 & 0.239523 & 0.495512 \\
\hline $\mathrm{C}$ & -2.812887 & 0.702565 & -0.255928 \\
\hline $\mathrm{C}$ & -4.052416 & 0.025022 & 0.278009 \\
\hline $\mathrm{C}$ & -4.898282 & -0.702781 & -0.599210 \\
\hline $\mathrm{C}$ & -4.593654 & -0.751190 & -1.968675 \\
\hline $\mathrm{C}$ & -3.478455 & -0.101568 & -2.534383 \\
\hline $\mathrm{C}$ & -2.635618 & 0.584571 & -1.727803 \\
\hline $\mathrm{C}$ & -4.413293 & 0.119520 & 1.611461 \\
\hline $\mathrm{C}$ & -5.566429 & -0.503810 & 2.074724 \\
\hline $\mathrm{C}$ & -6.392929 & -1.240008 & 1.221402 \\
\hline $\mathrm{C}$ & -6.063548 & -1.335689 & -0.106360 \\
\hline $\mathrm{H}$ & 1.743611 & -1.157084 & -1.365248 \\
\hline $\mathrm{H}$ & 2.484093 & 1.236617 & -1.783148 \\
\hline $\mathrm{H}$ & -0.029334 & -2.695804 & -0.640835 \\
\hline $\mathrm{H}$ & 2.500952 & 3.612763 & -1.143731 \\
\hline $\mathrm{H}$ & -1.976950 & -1.913766 & 0.562309 \\
\hline $\mathrm{H}$ & 0.470517 & 4.443074 & -0.104121 \\
\hline $\mathrm{H}$ & -1.692511 & 0.361983 & 1.550925 \\
\hline $\mathrm{H}$ & -1.211974 & 2.915887 & 0.821345 \\
\hline $\mathrm{H}$ & -2.911914 & 1.766417 & -0.044712 \\
\hline $\mathrm{H}$ & -1.780772 & 1.077615 & -2.148584 \\
\hline $\mathrm{H}$ & -3.817321 & 0.685292 & 2.299461 \\
\hline $\mathrm{H}$ & -5.829316 & -0.413130 & 3.110446 \\
\hline $\mathrm{H}$ & -3.312406 & -0.159142 & -3.589941 \\
\hline $\mathrm{H}$ & -5.250693 & -1.300272 & -2.618546 \\
\hline $\mathrm{H}$ & -6.691714 & -1.882155 & -0.783452 \\
\hline $\mathrm{H}$ & -7.275418 & -1.711714 & 1.601578 \\
\hline $\mathrm{H}$ & 3.865890 & -1.626097 & 2.907153 \\
\hline $\mathrm{H}$ & 2.689385 & 0.427188 & 3.537175 \\
\hline $\mathrm{H}$ & 2.344172 & 2.189581 & 1.877790 \\
\hline $\mathrm{H}$ & 3.995341 & 2.027460 & 0.037013 \\
\hline $\mathrm{H}$ & 4.647752 & 0.502587 & -2.096071 \\
\hline $\mathrm{H}$ & 5.923663 & -1.519820 & -2.545291 \\
\hline $\mathrm{H}$ & 6.161791 & -3.284767 & -0.846200 \\
\hline $\mathrm{H}$ & 5.118252 & -2.987778 & 1.362538 \\
\hline
\end{tabular}

Fig10 : $\mathrm{Naph}_{3}{ }^{2+}-\mathrm{II}$

$54 \mathrm{E}(\mathrm{RHF} / 6-31 \mathrm{G})=-1149.02107836 \mathrm{a} \cdot \mathrm{u}$.

\begin{tabular}{|c|c|c|c|}
\hline $\mathrm{C}$ & 6.105491 & -0.158903 & -1.189385 \\
\hline $\mathrm{C}$ & 4.916791 & -0.584361 & -0.549832 \\
\hline $\mathrm{C}$ & 4.088590 & 0.353551 & 0.120017 \\
\hline $\mathrm{C}$ & 4.488553 & 1.679056 & 0.154017 \\
\hline $\mathrm{C}$ & 5.663975 & 2.075106 & -0.472839 \\
\hline $\mathrm{C}$ & 6.473971 & 1.160974 & -1.153894 \\
\hline $\mathrm{C}$ & 4.572835 & -1.944222 & -0.526603 \\
\hline $\mathrm{C}$ & 3.440056 & -2.441674 & 0.149073 \\
\hline $\mathrm{C}$ & 2.614969 & -1.574000 & 0.78014 \\
\hline $\mathrm{C}$ & 2.815912 & -0.103719 & 0.786760 \\
\hline $\mathrm{C}$ & 1.518670 & 0.634521 & 0.185108 \\
\hline $\mathrm{C}$ & 0.281229 & 0.372354 & 1.04313 \\
\hline $\mathrm{C}$ & -0.808209 & -0.475698 & 0.50706 \\
\hline
\end{tabular}




\begin{tabular}{|c|c|c|c|}
\hline C & -0.777208 & -0.839043 & -0.781142 \\
\hline $\mathrm{C}$ & 0.284011 & -0.410393 & -1.691544 \\
\hline C & 1.333562 & 0.282034 & -1.266920 \\
\hline C & 0.173227 & 0.927201 & 2.256643 \\
\hline C & -0.974060 & 0.685793 & 3.126941 \\
\hline $\mathrm{C}$ & -1.949796 & -0.135792 & 2.763403 \\
\hline C & -1.929045 & -0.907523 & 1.460490 \\
\hline C & -3.372643 & -0.954351 & 0.815401 \\
\hline $\mathrm{C}$ & -3.829356 & 0.260023 & 0.038287 \\
\hline C & -4.585932 & 0.082793 & -1.155093 \\
\hline $\mathrm{C}$ & -4.843110 & -1.206833 & -1.634243 \\
\hline $\mathrm{C}$ & -4.369068 & -2.374519 & -0.999998 \\
\hline C & -3.647784 & -2.254145 & 0.137742 \\
\hline $\mathrm{C}$ & -5.084671 & 1.205739 & -1.861102 \\
\hline $\mathrm{C}$ & -4.845585 & 2.472868 & -1.402049 \\
\hline C & -4.104483 & 2.637091 & -0.227233 \\
\hline C & -3.605405 & 1.552402 & 0.482937 \\
\hline $\mathrm{H}$ & -1.558866 & -1.438283 & -1.204487 \\
\hline $\mathrm{H}$ & -1.729338 & -1.941576 & 1.746776 \\
\hline $\mathrm{H}$ & 0.179594 & -0.653995 & -2.730959 \\
\hline $\mathrm{H}$ & -2.772173 & -0.332572 & 3.426194 \\
\hline $\mathrm{H}$ & 2.086200 & 0.617514 & -1.954086 \\
\hline $\mathrm{H}$ & -1.001988 & 1.178202 & 4.078971 \\
\hline $\mathrm{H}$ & 1.755663 & 1.688247 & 0.257531 \\
\hline $\mathrm{H}$ & 0.940561 & 1.583331 & 2.624583 \\
\hline $\mathrm{H}$ & 2.850240 & 0.208603 & 1.829284 \\
\hline $\mathrm{H}$ & 1.753766 & -1.943147 & 1.301915 \\
\hline $\mathrm{H}$ & 3.904750 & 2.411492 & 0.675117 \\
\hline $\mathrm{H}$ & 5.958121 & 3.105448 & -0.429022 \\
\hline $\mathrm{H}$ & 3.252526 & -3.495163 & 0.159745 \\
\hline $\mathrm{H}$ & 5.215387 & -2.642219 & -1.031982 \\
\hline $\mathrm{H}$ & 6.719571 & -0.880915 & -1.692661 \\
\hline $\mathrm{H}$ & 7.373988 & 1.490875 & -1.630308 \\
\hline $\mathrm{H}$ & -5.426094 & -1.312122 & -2.531078 \\
\hline $\mathrm{H}$ & -4.589540 & -3.334140 & -1.419448 \\
\hline $\mathrm{H}$ & -3.290287 & -3.138082 & 0.632758 \\
\hline $\mathrm{H}$ & -4.046383 & -1.002542 & 1.682147 \\
\hline $\mathrm{H}$ & -3.050090 & 1.728998 & 1.378723 \\
\hline $\mathrm{H}$ & -3.918383 & 3.627568 & 0.139597 \\
\hline $\mathrm{H}$ & -5.222793 & 3.326725 & -1.925945 \\
\hline $\mathrm{H}$ & -5.657357 & 1.049773 & -2.755145 \\
\hline
\end{tabular}

Fig11 : Bz-Naph-Bz ${ }^{2+}$

$42 \mathrm{E}(\mathrm{RHF} / 6-31 \mathrm{G})=-843.783647601 \mathrm{a} \cdot \mathrm{u}$.

$\begin{array}{lrrr}\text { C } & 2.691352 & 1.411874 & -1.385399 \\ \mathrm{C} & 2.742656 & 1.421820 & 0.093283 \\ \mathrm{C} & 4.112991 & 1.430307 & 0.659039 \\ \mathrm{C} & 5.222516 & 1.455435 & -0.120369 \\ \mathrm{C} & 5.072227 & 1.457957 & -1.523865 \\ \mathrm{C} & 3.811892 & 1.438633 & -2.152483 \\ \mathrm{C} & 1.823653 & 0.292050 & 0.760570 \\ \mathrm{C} & 0.347737 & 0.504548 & 0.411665 \\ \mathrm{C} & -0.347742 & -0.504547 & -0.411669 \\ \mathrm{C} & 0.300039 & -1.609142 & -0.807103 \\ \mathrm{C} & 1.661766 & -1.913951 & -0.370621 \\ \mathrm{C} & 2.355225 & -1.067745 & 0.380716 \\ \mathrm{C} & -0.300044 & 1.609143 & 0.807100\end{array}$




$\begin{array}{lrrr}\mathrm{C} & -1.661771 & 1.913952 & 0.370619 \\ \mathrm{C} & -2.355230 & 1.067747 & -0.380718 \\ \mathrm{C} & -1.823658 & -0.292047 & -0.760576 \\ \mathrm{C} & -2.742661 & -1.421818 & -0.093294 \\ \mathrm{C} & -4.113002 & -1.430289 & -0.659035 \\ \mathrm{C} & -5.222519 & -1.455421 & 0.120386 \\ \mathrm{C} & -5.072214 & -1.457962 & 1.523880 \\ \mathrm{C} & -3.811873 & -1.438653 & 2.152485 \\ \mathrm{C} & -2.691341 & -1.411891 & 1.385388 \\ \mathrm{H} & 1.949161 & 0.435774 & 1.829290 \\ \mathrm{H} & 3.332348 & -1.329726 & 0.740445 \\ \mathrm{H} & 0.185859 & 2.335608 & 1.432437 \\ \mathrm{H} & -2.075445 & 2.864952 & 0.642845 \\ \mathrm{H} & 2.075438 & -2.864952 & -0.642844 \\ \mathrm{H} & -0.185863 & -2.335607 & -1.432440 \\ \mathrm{H} & -1.949165 & -0.435767 & -1.829297 \\ \mathrm{H} & -3.332354 & 1.329727 & -0.740445 \\ \mathrm{H} & -4.206639 & -1.435204 & -1.729327 \\ \mathrm{H} & -6.203258 & -1.477492 & -0.307761 \\ \mathrm{H} & -5.954491 & -1.476705 & 2.136349 \\ \mathrm{H} & -3.753373 & -1.443915 & 3.221098 \\ \mathrm{H} & -1.722307 & -1.397995 & 1.845034 \\ \mathrm{H} & -2.285720 & -2.367363 & -0.407804 \\ \mathrm{H} & 4.206616 & 1.435236 & 1.729333 \\ \mathrm{H} & 6.203250 & 1.477518 & 0.307788 \\ \mathrm{H} & 5.954510 & 1.476697 & -2.136325 \\ \mathrm{H} & 3.753404 & 1.443881 & -3.221098 \\ \mathrm{H} & 1.722323 & 1.397967 & -1.845056 \\ \mathrm{H} & 2.285704 & 2.367365 & 0.407778 \\ & & & \end{array}$

\begin{tabular}{lrrr} 
Fig12 : Bz-Bz-Naph-Bz-Bz & \\
66 E (RHF $/ 6-31 G)=$ & -1304.99000481 & \multicolumn{1}{l}{ a.u. } \\
& & & \\
C & 8.049165 & -1.027910 & -0.365311 \\
C & 6.879927 & -0.301759 & -0.900491 \\
C & 6.902232 & 1.153996 & -0.668018 \\
C & 7.927786 & 1.773513 & -0.023771 \\
C & 8.999857 & 0.999046 & 0.453063 \\
C & 9.062396 & -0.399355 & 0.284252 \\
C & 5.490478 & -0.969995 & -0.450519 \\
C & 5.242348 & -0.771591 & 1.022190 \\
C & 4.138619 & -0.219909 & 1.503837 \\
C & 2.997812 & 0.286724 & 0.660931 \\
C & 3.262715 & 0.095973 & -0.807305 \\
C & 4.363500 & -0.450448 & -1.303009 \\
C & 1.627952 & -0.357398 & 1.109341 \\
C & 0.438833 & 0.365534 & 0.467626 \\
C & -0.438833 & -0.365526 & -0.467625 \\
C & -0.215200 & -1.664393 & -0.709951 \\
C & 0.818466 & -2.423841 & -0.010085 \\
C & 1.642339 & -1.842354 & 0.852583 \\
C & 0.215198 & 1.664401 & 0.709953 \\
C & -0.818468 & 2.423849 & 0.010088 \\
C & -1.642341 & 1.842361 & -0.852580 \\
C & -1.627953 & 0.357406 & -1.109340 \\
C & -2.997812 & -0.286719 & -0.660930 \\
C & -4.138619 & 0.219912 & -1.503838 \\
C & -5.242350 & 0.771592 & -1.022192
\end{tabular}




$\begin{array}{rrrr}\mathrm{C} & -5.490482 & 0.969998 & 0.450517 \\ \mathrm{C} & -4.363503 & 0.450453 & 1.303008 \\ \mathrm{C} & -3.262717 & -0.095966 & 0.807306 \\ \mathrm{C} & -6.879930 & 0.301759 & 0.900489 \\ \mathrm{C} & -6.902227 & -1.153998 & 0.668027 \\ \mathrm{C} & -7.927775 & -1.773525 & 0.023781 \\ \mathrm{C} & -8.999848 & -0.999066 & -0.453063 \\ \mathrm{C} & -9.062395 & 0.399336 & -0.284263 \\ \mathrm{C} & -8.049169 & 1.027901 & 0.365298 \\ \mathrm{H} & 1.575262 & -0.196860 & 2.184344 \\ \mathrm{H} & 2.372381 & -2.420260 & 1.386233 \\ \mathrm{H} & 0.829132 & 2.199677 & 1.410670 \\ \mathrm{H} & -0.873659 & 3.480239 & 0.190501 \\ \mathrm{H} & 0.873655 & -3.480232 & -0.190498 \\ \mathrm{H} & -0.829134 & -2.199670 & -1.410667 \\ \mathrm{H} & -1.575263 & 0.196868 & -2.184342 \\ \mathrm{H} & -2.372384 & 2.420267 & -1.386230 \\ \mathrm{H} & -4.024898 & 0.120267 & -2.568856 \\ \mathrm{H} & -6.002090 & 1.124619 & -1.696814 \\ \mathrm{H} & -5.627833 & 2.028829 & 0.657232 \\ \mathrm{H} & -4.466281 & 0.580511 & 2.366063 \\ \mathrm{H} & -2.488388 & -0.418114 & 1.477808 \\ \mathrm{H} & -2.890908 & -1.352677 & -0.850090 \\ \mathrm{H} & 4.024899 & -0.120266 & 2.568856 \\ \mathrm{H} & 6.002088 & -1.124620 & 1.696811 \\ \mathrm{H} & 5.627827 & -2.028827 & -0.657236 \\ \mathrm{H} & 4.466277 & -0.580504 & -2.366064 \\ \mathrm{H} & 2.488386 & 0.418123 & -1.477806 \\ \mathrm{H} & 2.890909 & 1.352683 & 0.850092 \\ \mathrm{H} & -6.064416 & -1.719011 & 1.028420 \\ \mathrm{H} & -7.927568 & -2.832450 & -0.130830 \\ \mathrm{H} & -9.804077 & -1.491659 & -0.966949 \\ \mathrm{H} & -9.903915 & 0.942460 & -0.661491 \\ \mathrm{H} & -8.081777 & 2.090866 & 0.516242 \\ \mathrm{H} & -6.892005 & 0.452057 & 1.987841 \\ \mathrm{H} & 6.064423 & 1.719015 & -1.028403 \\ \mathrm{H} & 7.927584 & 2.832438 & 0.130848 \\ \mathrm{H} & 9.804090 & 1.491631 & 0.966949 \\ \mathrm{H} & 9.903916 & -0.942486 & 0.661472 \\ \mathrm{H} & 6.891998 & -2.090875 & -0.516264 \\ & & -0.452048 & -1.987845\end{array}$




\section{Complete or additional references}

[7] (k) Janhke, T.; Czasch, A.; Schössler, S.; Knapp, A.; Käsz, M.; Titze, J.; Wimmer, C.; Kreidi, K.; Grisenti, R.E.; Staudte, A.; Jagutzki, O.; Hergenhahn, U.; Schmidt-Böcking, H.; Dörner, R.; Phys. Rev. Lett. 2004, 93, 163401; (1) Öhrwall, G.; Tchaplyguine, M.; Luindwall, M.; Feifel, R.; Bergersen, H.; Rander, T.; Limdblad, A.; Schulz, J.; Peredkovb, S.; Bart, S.; Marburger, S.; Hergenhahn, U.; Svensson, S.; Björneholm, Phys. Rev. Lett. 2004, 93, 173401.

[22] Frisch, M.J.; Trucks, G.W.; Schlegel, H.B.; Scuseria, G.E.; Robb, M.A.; Cheeseman, J.R.; Zakrzewski, V.G.; Montgomery Jr, J.A.; Stratmann, R.E.; Burant, J.C.; Dapprich, S.; Millam, J.M.; Daniels, A.D.; Kudin, K.N.; Strain, M.C.; Farkas, O.; Tomasi, J.; Barone, V.; Cossi, M.; Cammi, R.; Mennucci, B.; Pomelli, C.; Adamo, C.; Clifford, S.; Ochterski, J.; Petersson, G.A.; Ayala, P.Y.; Cui, Q.; Morokuma, K.; Malick, D.K.; Rabuck, A.D.; Raghavachari, K.; Foresman, J.B.; Cioslowski, J.; Ortiz, J.V.; Stefanov, B.B; Liu, G.; Liashenko, A.; Piskorz, P.; Komaromi, I.; Gomperts, R.; Martin, R.L.; Fox, D.J.; Keith, T.; Al-Laham, M.A.; Peng, C.Y.; Nanayakkara, A.; Gonzalez, C.; Challacombe, M.; Gill, P.M.W.; Johnson, B.G.; Chen, W.; Wong, M.W.; Andres, J.L.; Gonzalez, C.; Head-Gordon, M.; Replogle E.S.; Pople, J.A.; Gaussian98, revision A.7, Gaussian, Inc., Pittsburg, PA, 1998.

[49] J.-M. André, J. Delhalle, J.-L. Brédas, Quantum Chemistry Aided Design of Organic Polymers, World Scientific, Singapore, 1991.

[50] (a) Suhai, S.; Chem. Phys. Lett. 1983, 96, 619; (b) Suhai, S.; Phys. Rev. B 1983, 27, 3506; (c) Miao, M.S.; Van Camp, P.E.; van Doren, V.E.; Ladik, J.J.; Mintimre, J.W.; J. Chem. Phys. 1998, 109, 9623; (d) Kwon, O.; McKee, M.L.; J. Phys. Chem. B 2000, 104, 1686; (e) Kwasniewski, S.P.; Deleuze, M.S.; J.-P. François, Int. J. Quantum Chem. 2000, $80,672.9$ 